\title{
Corela
}

Cognition, représentation, langage

$16-2 \mid 2018$

Vol. $16, n^{\circ} 2$

\section{Más et ses « marqueurs du standard » en espagnol : de que.}

Approche des signifiants en chronosyntaxe interlocutive

\section{Marine Poirier}

\section{OpenEdition}

\section{Journals}

Electronic version

URL: http://journals.openedition.org/corela/6910

DOI: $10.4000 /$ corela.6910

ISSN: 1638-573X

\section{Publisher}

Cercle linguistique du Centre et de l'Ouest - CerLICO

\section{Electronic reference}

Marine Poirier, "Más et ses « marqueurs du standard » en espagnol : de que. », Corela [Online], 16-2 I

2018, Online since 05 December 2018, connection on 10 December 2020. URL : http://

journals.openedition.org/corela/6910; DOI : https://doi.org/10.4000/corela.6910

This text was automatically generated on 10 December 2020 .

\section{c) (i)(2)}

Corela - cognition, représentation, langage est mis à disposition selon les termes de la licence Creative Commons Attribution - Pas d'Utilisation Commerciale - Partage dans les Mêmes Conditions 4.0 International. 


\section{Más et ses « marqueurs du standard » en espagnol : de que.}

Approche des signifiants en chronosyntaxe interlocutive

\section{Marine Poirier}

\section{Introduction}

1 Lorsque les grammaires de l'espagnol soulignent l'impossibilité de construire ou de rencontrer des structures du type suivant :

(1) *Carlos se tomó más de tres cervezas que Alfredo. (NGLE 45.7b) ${ }^{1}$

ce qui est mis en évidence, c'est la façon dont les segments introduits par de et par que entrent en contradiction et en concurrence l'un avec l'autre dans une phrase fictive de ce type. En appréhendant la phrase dans la séquentialité de l'enchaînement de ses signifiants - dans sa chrono-syntaxe ${ }^{2}$ (Macchi 2000 et sq.) -, más, déclencheur de comparaison dans cette phrase, ouvre un espace de «transitivité phrastique » (au sens de Macchi $2006: 130^{3}$ ) en ce qu'il proacte la recherche ou l'attente d'un possible repère ${ }^{4}$. Si la survenance de que comme marqueur du standard (que Alfredo) est incongrue aux yeux de l'interprétant, c'est qu'elle n'était plus attendue; ou, pour être tout à fait exacte, qu'elle ne figurait plus parmi le « champ des possibles » à l'instant de sa survenance. C'est donc que cet espace de transitivité ouvert par más a été comblé dans l'antécédence phrastique de que : en l'occurrence, par de et l'empan phrastique que ce dernier permet d'introduire ( tres cervezas). En prenant ainsi la phrase dans sa séquentialité :

* t0 Carlos se tomó t1 más t2 de t3 tres cervezas t4 \# que $t 5$ Alfredo tf \#.

Figure 1.5

3 de fait, en $\mathrm{t} 4$, le repère répondant à más ayant été posé en t2-t4 et annoncé par de, la construction peut s'achever (\#) à l'entière satisfaction du locuteur comme de l'interlocuteur; que intervenant en $\mathrm{t} 4$-t5 paraît rééditer un repère rendu périmé par le 
précédent à l'instant de sa survenance. De et que fonctionnent alors en dialectique avec más dans le cadre du fonctionnement de ce dernier comme déclencheur de comparaison.

Ces deux signifiants, toutefois, répondent chacun à leur manière à más. Si la différence entre Carlos se tomó más de tres cervezas et Carlos se tomó más cerverzas que Alfredo semble annoncer une répartition relativement simple entre de et que (différence de placement de de et de que, segment introduit sensiblement différent syntaxiquement dans le cas de de et dans celui de que), les choses se compliquent dès lors que l'on observe dans les discours authentiques de nombreux cas attestés d'alternance apparemment libre, dans des cotextes syntaxiques similaires. Ainsi, on trouvera aussi bien más de tres que más que tres:

(2) El PSOE mantiene una ventaja de más de tres puntos sobre el PP

(https://www.20minutos.es/noticia/343382/0/mantiene/ventaja/puntos/, consulté le 4 novembre 2018. C'est nous qui soulignons)

(3) Llega a El Plantío uno de los partidos más esperados de la temporada, visita el coliseo blanquinegro el Racing de Santander, uno de los equipos fuertes del grupo. En juego más que tres puntos, un partido para recuperar la senda de la victoria, acercarse a los puestos de Copa y seguir luchando hasta el final por el playoff. (http://www.burgoscf.es/ficha/Z460E3FC9-0BA9-02ED-D93B0C3D514E84EC/masque-tres-puntos, consulté le 8 avril 2018. C'est nous qui soulignons) ${ }^{6}$

Ou encore, más de lo habitual ou más que lo habitual :

(4) María Elena pasa otras dos semanas sin salir, sin peinarse, sin lavarse. Cree que se va a morir. Y está conforme. Higinio tarda más de lo habitual en volver de la ciudad de México, pero vuelve con un niño.

(CORPES XXI, José Montelongo, Quincalla, México 2004. C'est nous qui soulignons)

(5) En este programa improvisado (aún más que lo habitual) damos unas cuántas recomendaciones para ver en Netflix, también hablamos un poco sobre el manga de Dr. Stone.

(Google, https://itunes.apple.com/us/podcast/mal-vivir/id1219873916?mt=2 , consulté le 8 juin 2018. C'est nous qui soulignons) ${ }^{7}$

6 Et même des cas comme les suivants, où l'opposition de que trouve le moyen de se manifester sous la forme más que más de lo que:

(6) Cada traslado lo realizaban en peores condiciones. Entonces se volvió una niña traviesa y respondona, mentía más de lo que hablaba.

(Reina Roffé, El otro amor de Federico : Lorca en Buenos Aires. Barcelona : Plaza \& Janés, 2009 , p. 283. C'est nous qui soulignons)

(7) Blanca se encargó de los papeles modificados, y Elsa grande, a la que los adultos consideraban más sensata y de la que no sospechaban, porque Blanca mentía más que hablaba, trató de convencer a los padres para que las dejaran ir.

(Espido Freire, Melocotones helados. Barcelona : Planeta, 1999 ; cité par NGLE 45.60.

C'est nous qui soulignons) ${ }^{8}$

Qu'est-ce qui motive le choix de l'emploi de de ou de que en position de répondant à más chez un locuteur hispanophone? Quel effet produit l'emploi de de, quel effet l'emploi de que ? Est-il possible de rendre raison de la multiplicité des cas d'emploi de de par un même effet commun, de la multiplicité des cas d'emploi de que par un même effet commun, et de rendre ainsi compte de l'alternance más de más que par deux effets invariants s'opposant l'un à l'autre?

8 La question de l'alternance ou de la répartition de / que en position de «marqueur du standard » et de solveur d'une structure de comparaison ouverte par más ou menos figure en très bonne place parmi les problèmes les plus complexes et les plus débattus dans la littérature sur la comparaison, faisant partie de celles qui font dire à Gutiérrez Ordóñez (1994a : 9-10), jouant sur un dicton de la sagesse populaire pour y ajouter un grain de sel 
de grammairien, que « las comparaciones son odiosas [...], también en gramática » ${ }^{9}$. C'est cette question que l'on soulève à nouveau ici, pour la soumettre cette fois à une approche guidée par une conception de la parole humaine en tant que technique vocale de production $d u$ sens: l'enchaînement vocal qu'est la phrase y est conçu comme une dynamique permettant de guider pas à pas, signifiant après signifiant, la construction du sens linguistique chez l'allocutaire (à qui on s'adresse) comme chez le locuteur (qui, réflexivement, s'entend penser en temps réel). Le sens linguistique n'est donc pas pensé comme une représentation autonome, préalablement conçue par un centre cognitif locutif seul et qui serait encodée par des signes puis décodée par un interlocuteur, mais comme un événement à façonner en temps réel via un enchaînement de signifiants. Cette approche épistémologique, qui se situe dans un paradigme énactivisant en linguistique (Bottineau 2017a), se décline en plusieurs modèles théoriques et méthodologiques dont l'un des enjeux actuels ${ }^{10}$ est leur mise en dialogue et en synergie; parmi ceux-là, la théorie de la relation interlocutive (Douay \& Roulland 2014), la chronosyntaxe (Macchi 2000 et sq.) et la lecture du geste signifiant considéré pour l'effet qu'il est susceptible de produire sur les interprétants (avec notamment la cognématique, Bottineau 2000 et sq.), seront ici articulés. Chacun sera présenté en détails dans le corps de l'étude.

Dans ce paradigme, c'est en termes de cadre réceptif dans l'interlocution qu'un invariant associé à más de d'un côté, et un attaché à más que de l'autre, seront ici formulés. Il est au moins deux usages du terme « interlocution » : l'un général et pré-théorique, désignant le dialogue et la co-présence de deux personnes réelles engagées dans un échange verbal; l'autre particulier et renvoyant au modèle théorique de Douay \& Roulland (2014), la théorie de la relation interlocutive (TRI), avec sa terminologie et son appareil conceptuel propre. On partira ici dans un premier temps du sens le plus général du terme « interlocution" pour spécialiser ensuite cette étude dans son usage le plus spécifique, dès lors que l'on proposera, en observant les signifiants más de más que dans leur matérialité, d'inscrire leur contribution opératoire à la dynamique de construction du sens dans une élaboration progressive de la relation langagière entre pôle locutif et pôle allocutif. Cette contribution opératoire reposera ainsi respectivement sur la cognématique ("matérialité » des signifiants), la chronosyntaxe ("progressivité » de l'élaboration de la phrase), et la TRI («relation langagière » constitutive de deux pôles locutif et allocutif). C'est la synthèse de ces modèles qui permettra ici de tenter de saisir les dyptiques más de más que. Quel est l'effet produit sur l'interprétant - et, partant, sur la relation qui s'établit entre locuteur et allocutaire par le biais de l'interface qu'est la parole et que sont les signifiants - par les signifiants de et que agissant en répondants à más ayant proacté leur survenance ? En quoi ces effets diffèrent-ils et pourquoi sont-ils susceptibles de donner lieu, à l'interprétation de l'énoncé entièrement constitué, à des constructions syntaxiques jugées totalement disparates par un grammairien ou un linguiste? Telles sont les questions que l'on soumet à ce regard méthodologique, qui se veut phénoménologiquement en acte.

10 Un état raisonné du problème (section 1) permettra de faire le point sur les différents types d'approches de la répartition de $\sim$ que dans les nombreux travaux existants, en passant par une typologie des effets de sens relevés par la littérature; de cette typologie, on ne discutera pas la pertinence interne, mais on soulèvera la question de la démarche implicite qui sous-tend son mode d'élaboration et on questionnera certaines conclusions auxquelles elle donne lieu. Ce questionnement conduira à la proposition d'une démarche alternative, par laquelle on proposera (section 2) la formulation pour más $\sim$ de et más que 
d'une contribution en termes de cadre réceptif invariant (Douay \& Roulland 2014 : 179-180) installé par les signifiants dans la relation interlocutive. On explorera en dernier lieu (section 3) la façon dont la systématique interlocutive proposée permet de rendre raison des différents effets de sens détectés par les typologies grammaticales traditionnelles.

\section{La répartition de $\sim$ que. État de la question}

\subsection{Une vaine recherche de déterminisme par le cotexte syntaxique}

11 Sans doute serait-il tentant en premier lieu, en particulier dans une perspective didactique (Mello 1977, Sáenz 1940, ou les grammaires de l'espagnol pour apprenants non hispanophones), d'essayer de parvenir à dresser pour la paire de que une stricte distribution complémentaire dépendant mécaniquement du cotexte syntaxique. C'est l'entreprise dans laquelle se lancent certains travaux, qui font reposer une telle distribution complémentaire sur la nature de l'élément suivant immédiatement de ou que (Plann 1984, Bedel 1997) ou au contraire sur la nature (Gerboin \& Leroy 1991) ou la fonction (Prytz 1979) de l'antécédent; mais la tentation est vite déçue par la réalité du discours, bien plus complexe qu'espéré, en particulier lorsqu'on se penche non plus sur des exemples forgés afin d'illustrer une étiquette, mais sur des énoncés authentiques « à l'état sauvage ». Pour prendre un seul exemple, la proposition d'utiliser systématiquement de lorsque le $2^{\mathrm{e}}$ terme de la comparaison est une proposition et que lorsqu'il s'agit d'une syntagme nominal (Plann 1984) ou de lorsque le $2^{\mathrm{e}}$ terme est un verbe conjugué et que lorsqu'il n'en est pas un (Bedel 1997: 501-502) - sur le modèle Alberto parece más grande que Juan vs. Alberto parece más grande de lo que era antes - est vite découragée par des exemples comme le suivant, déjà cité en introduction :

(7) Blanca mentía más que hablaba.

(Espido Freire, Melocotones helados, 1999 ; cité par NGLE 45.60). ${ }^{11}$

12 La multiplication des critères de choix est parfois la solution adoptée (Bolinger 1950), les uns faisant écho à ceux des autres pour en ajouter de nouveaux dès lors que l'insuffisance des premiers seuls a été constatée ; on n'est plus très loin, alors, de l'aveu indirect d'une impuissance ou d'un échec à repérer une distinction simple et économique, et de l'obligation de se rendre à un listage quasiment au « cas par cas » de la multiplicité des usages (comme le fait remarquer Sáenz 1940).

13 S'il semble vain de rechercher un déterminisme dû à l'entourage grammatical, il est toutefois au moins un point qui semble acquis : en espagnol contemporain, de en position de répondant à más présente des enchaînements plus contraints que ceux de que. On pourra les regrouper de la manière suivante (Gutiérrez Ordóñez 1992) : de suivi de lo (suivi d'une relative ou d'un adjectif ou participe passé, exemples 8 et 9) ou de suivi d'un numéral (exemple 10 ci-dessous). Cependant, si, en espagnol contemporain toujours, certains contextes syntaxiques avec que sont bloquants pour de (on y reviendra section 3.4), tous les cas précédemment mentionnés où intervient de peuvent voir l'intervention de que en lieu et place de ce dernier. En d'autres termes, dès lors que de est susceptible d'intervenir, le locuteur a bien le choix entre de et que:

(8a) Esto es más bonito de lo que yo había imaginado.

(8b). Esto es más bonito que lo que yo había imaginado.

(NGLE Manual : 45.2.6e)

(9a) He traído más de lo estrictamente necesario. 
(9b) He traído más que lo estrictamente necesario.

(NGLE : 45.6u)

(10a) Se discutieron más de sesenta ponencias.

(10b) Se discutieron más que sesenta ponencias. ${ }^{12}$

(Sáez \& Sánchez López 2013 : 71)

Dans les travaux où la répartition más de $\sim$ más que est reconnue comme étant irréductible à une distribution complémentaire selon un strict critère syntaxique mécanique, et où repérée la possibilité de choix entre de et que, ce choix (embarrassant) donne lieu à diverses explications. Est parfois évoquée une volonté d'éviter la cacophonie que pourrait supposer l'usage de que lorsque celui-ci doit être convoqué immédiatement après dans la phrase - de lo que serait ainsi préféré à que lo que pour des raisons d'euphonie (explication proposée par Bello 1847, reprise par Solé 1982 ; que Spaulding 1962, notamment, s'attache à réfuter). Mais là aussi, l'observation du discours montre nombre d'énoncés où en aucun cas la présence de l'un ou de l'autre signifiant dans la suite de l'énoncé ne bloque son usage comme solveur de la transitivité phrastique ouverte par más (exemple $8 \mathrm{~b}$ ci-dessus, où l'enchaînement que lo que ne pose pas de problème).

\subsection{La recherche d'une différence sémantique ou pragmatique entre más que et más de : les difficultés d'une tentative de régulation par la délimitation de catégories strictes (comparaison propre vs. autres effets de sens)}

La question s'impose alors: dans ces cas où le locuteur a le choix, où le processus phrastique est potentiellement le même jusqu'à la survenance de de ou de que à un instant $t$ de son déroulement, le recours à l'un ou à l'autre des deux signifiants peut-il provoquer strictement le même effet? C'est à la recherche d'une différence sémantique ou pragmatique que s'attache une dernière série de travaux (Gutiérrez Ordóñez 1994ab, Ander Media à paraître, Rojas Nieto 1990). Considérons par exemple, dans un premier temps, la différence entre (8a) et (8b) ci-dessus. En (8b), sont comparées deux entités : l'une $x$ désignée par esto, l'autre $y$ désignée par lo dont il est précisé que yo había imaginado ; il est dit de l'entité $x$ « esto » qu'elle est plus belle que l'entité $y$ « que yo había imaginado ». En situation vécue, on imaginera par exemple une conversation entre deux conjoints ayant prévu de placer un objet décoratif sur la table du salon; l'un des deux, rentrant le soir et découvrant une babiole déposée par sa moitié à l'endroit convenu, pourra déclarer esto es más bonito que lo que yo había imaginado, à savoir : cette babiole est plus jolie que cette autre que, moi, j'aurais imaginée à cet endroit. En (8a), en revanche, une seule entité $x$ « esto » et pas d'entité $y$ : le degré de beauté de $x$ n'est pas comparé à celui d'une autre entité, mais simplement confronté à un point de référence (lo que yo había imaginado) qui correspond à une attente. En situation vécue, le couple précédemment évoqué pourrait débarquer sur un lieu de vacances et l'un des deux, s'extasier : esto es más bonito de lo que yo había imaginado, à savoir, ce lieu tel que je le vois aujourd'hui est encore plus beau que ce même lieu tel que je me l'étais figuré avant d'arriver. Pareillement, más de sesenta ponencias en (10a) déclare que la quantité de communications présentées au colloque est supérieure à un point de référence introduit par de et qui est sesenta.

16 Ceci étant posé, le débat semble alors se cristalliser sous la plume des uns et des autres autour d'une tentative de délimitation entre ce qui tient réellement de la comparaison et ce qui tiendrait d'une autre catégorie, dans laquelle ranger les structures en de posant un 
point de référence plus qu'une comparaison entre deux éléments. Et c'est là que les choses se corsent.

Car si un cas comme (10b), par exemple, est interprété à la lumière d'un énoncé tel que le suivant (11a) :

(11a) Velázquez pintó más cuadros que Las Meninas (NGLE 45.4n)

vs.

(11b) Velázquez pintó más cuadros que Murillo (NGLE 45.4n) ${ }^{13}$

où más cuadros que Las Meninas renvoie à des tableaux autres que ce dernier bien connu par opposition à (11b) où más cuadros que Murillo effectue une comparaison d'inégalité entre le nombre de tableaux peints par l'un et par l'autre -, alors, más que sesenta ponencias renverrait en fait à d'autres choses qui auraient été discutées en dehors des communications. Est-on toujours fondé dans ce cas à y coller l'étiquette « comparaison »? Et dans ce cas, ni (10a) avec más de ni (10b) avec más que n'auraient droit à cette étiquette? On le voit: les étiquettes collées aux structures en más ... que et más ... de semblent alors se démultiplier, dont on pourra dresser un rapide bilan dans le tableau 1 ci-dessous :

\begin{tabular}{|c|c|}
\hline QUE & $\mathrm{DE}$ \\
\hline 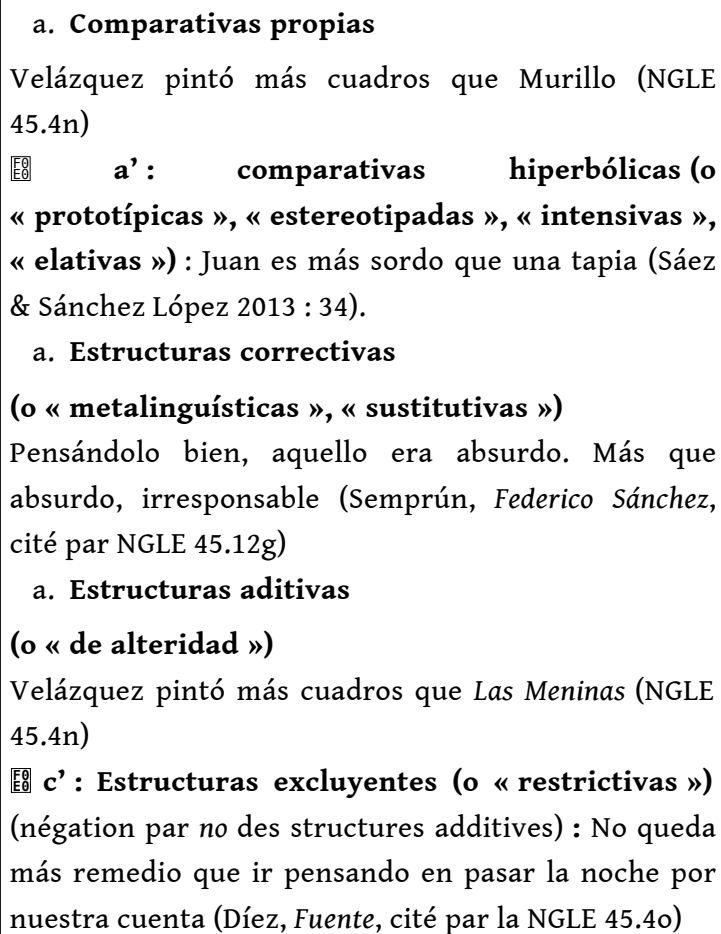 & 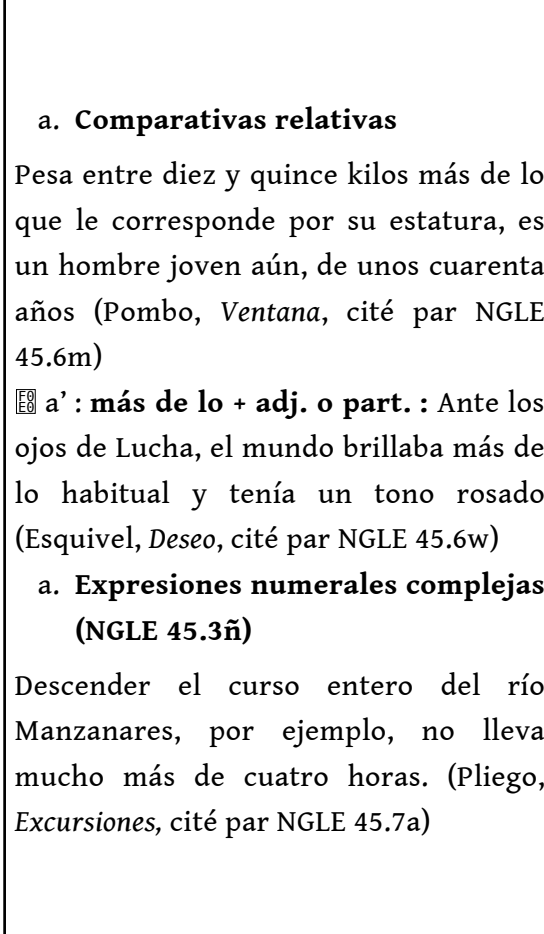 \\
\hline
\end{tabular}

Tableau 1. Tableau de synthèse de la littérature sur la question : typologie des structures en más de $\sim$ más que ${ }^{14}$

19 Cette typologie étant établie - certaines catégories faisant davantage consensus que d'autres -, les plus radicaux considèrent que seules les structures appelées "comparativas propias" (case que a.) dans le tableau ci-dessus méritent d'être considérées comme des structures comparatives, en ce qu'elles supposent la mise en parallèle de deux entités ${ }^{15}$ au regard d'un paramètre; les autres ne seraient 
qu'improprement considérées comme telles en ce qu'elles usurperaient le signifiant más des premières pour le corréler avec de ou (pire) pour le corréler avec un que lui-même usurpé aux structures « réellement » comparatives :

Estas comparativas "impropias", bautizadas en ocasiones como "pseudocomparativas" en la bibliografía, constituyen por tanto un caso llamativo de «usurpación formal» que obliga al investigador a proponer explícitos criterios demarcativos que permitan distinguirlas de las propias. (Sáez \& Sánchez López $2013: 31)^{16}$

Par exemple, pour Sáez del Álamo (1999: § 17.2.1, n68), «[e]l más de las construcciones aditivas no tiene con el elemento de grado otra relación que la de la pura homofonía $»^{17}$.

Seulement voilà : premièrement, on aura d'emblée remarqué le défaut d'étanchéité des différentes catégories - ainsi, certains exemples précédemment cités peuvent-ils, selon les cas, être classés dans une catégorie comme dans une autre : on pense à (10b), que l'on reprendra plus loin -, défaut révélateur de la difficulté, pour l'analyste, à faire entrer la diversité et la complexité du discours dans les cases d'une typologie abstraite fixe. Et surtout, deuxièmement, tenter d'établir une typologie stricte permettant de tracer les frontières d'une catégorie " comparaison » et débattre sur l'inclusion ou non de telle ou telle case de la typologie dans la catégorie en question, c'est oublier que toutes ces cases (structures comparatives, correctives, additives, etc.) ne sont que des élaborations réflexives et a posteriori par l'analyste. La tentative de régularisation du foisonnement du discours par des catégories réflexivement construites par l'analyste tend à créer des problèmes plus qu'à en résoudre; c'est le même type de constat que fait Goody - dans le cadre anthropologique qui est le sien, dans sa recherche sur les premières formes d'écriture (en l'occurrence, la liste) et l'impact de l'écriture sur le façonnage des structures cognitives -, ici au sujet de l'inclusion ou non d'un végétal dans la liste des fruits ou des légumes :

Certains types de listes [posent] d'intéressants problèmes de cognition. Sitôt la liste créée, on peut rassembler tous les mots désignant un légume, bref les décontextualiser et, ce faisant, établir une hiérarchie avec un commencement et une fin. La seule question que je me pose, c'est si je vais mettre tel ou tel végétal dans cette liste-ci ou dans une autre. [...]. La liste elle-même me pose une question de façon décontextualisée, alors que, dans une société orale, peut me chaut qu'une tomate soit un fruit dans tel contexte, un légume dans tel autre. Ainsi est-on conduit, avec la liste, à se poser des questions « scientifiques » qui n'avaient pas lieu d'être autrement. (Goody 1996 : 210)

L'élaboration d'une typologie d'effets de sens présente mutatis mutandis les mêmes conséquences. Elle a bien entendu le grand avantage de permettre de faire le bilan de différents effets de sens ; cependant, plus que de résoudre un problème - en l'occurrence, celui de l'alternance más de más que -, il a l'inconvénient d'en créer d'autres : car pour les expérients, on a bien là le signifiant más à chaque fois, et sans doute est-il légitime de se demander en quoi importe la case dans laquelle l'analyste rangera la production achevée. Dès lors, pour le linguiste qui tente de rendre compte de l'alternance más de más que et de la différence d'effet produit par le choix de de ou de que dans l'expérience vécue des locuteurs et interlocuteurs, les catégories proposées par la grammaire traditionnelle ne peuvent constituer une solution en elles-mêmes.

Le recours à la méthode chronosyntaxique permettra de souligner la distinction à faire entre, d'un côté, la reconnaissance de l'un de ces différents effets de sens ci-dessus cités 
et résultant de l'ensemble de l'énoncé, et d'un autre côté, l'effet produit en temps réel par le choix de de ou de que. Partant, elle orientera vers une autre démarche.

\subsection{La recherche d'une différence sémantique ou pragmatique entre más que et más de : pour une autre démarche}

On se placera donc à présent en position de récepteur d'une telle phrase ; l'interception de la phrase en plusieurs instants de sa construction dynamique (méthode chronosyntaxique), qui modélise les divers temps d'émergence du sens à la conscience d'un observateur procédural et épilinguistique, permet de saisir la progressivité de la construction du sens global de l'énoncé. Prenons l'exemple d'un énoncé dont la syntaxe est réputée dénuer la phrase de toute ambiguité quant à son classement dans la typologie ${ }^{18}$ :

(12) Al pie de las peñas estaban unas casas mal hechas, que más parecían ruinas de eficios que casas (Cervantes, Quijote, cité par NGLE 45.12f). ${ }^{19}$

Comme bien souvent (Macchi 2000 et sq.), ce n'est qu'en se plaçant à la toute fin de l'énoncé, en ayant à l'esprit sa totalité achevée, que l'observateur devient capable de distinguer un cas de « comparaison » d'un autre qui s'écarterait de la définition qu'il s'en donne; en l'occurrence, un cas « correctif ». L'analyse des signifiants appréhendés les uns à la suite des autres dans le temps de leur réception, en effet, suggère que le fonctionnement de más et de que ne diffère en rien à l'instant de leur survenance, et que l'effet « correctif » ou simplement « comparatif » est le fruit d'un acte d'interprétation de la phrase entière engendré par la prise en compte de la subséquence phrastique, le rangement de la phrase dans une catégorie ou une autre tenant alors à un commentaire réflexif de cet acte d'interprétation:

$$
\text { to } \ldots \text { casas mal hechas que } t 1 \text { más } t 2 \text { parecían ruinas t3 que t4 } \ldots
$$

Figure 2.

A la survenance de más, opérateur de surenchère marquant « une addition par rapport à un repère préalablement conçu " (Fortineau-Brémond 2012b: 80; Sicot-Domínguez $2010 \mathrm{ab})$, est proactée l'attente à la fois de l'élément de surenchère et du repère par rapport auquel il constitue une surenchère. L'empan t2-t3 (parecían ruinas) donne des éléments concernants le premier (éléments encore incomplets à cet instant de parole, puisqu'on verra ensuite qu'en fonction du repère - ici posé plus loin dans la subséquence

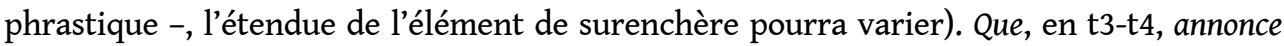
la survenance du repère ${ }^{20}$. A cet instant, la phrase pourrait encore parfaitement bifurquer de la manière suivante :

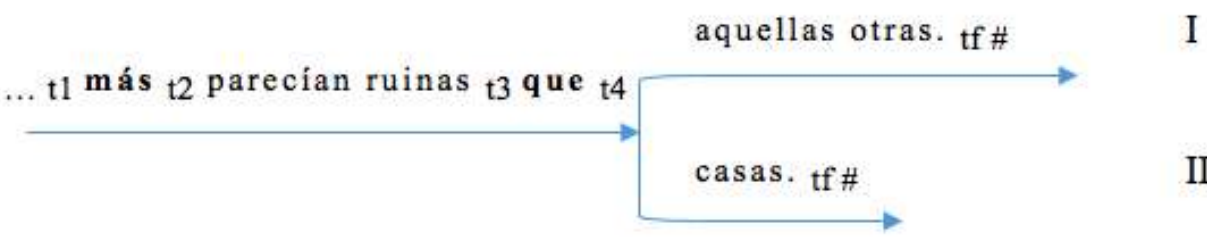

Figure 3. 
Dans le cas I, la survenance de aquellas otras fournit une entité comparante qui fait de casas une entité comparée, de parecer ruinas le paramètre de la comparaison. En II, la réédition du terme casas en $\mathrm{t} 4$-tf amène (à cet instant seulement) l'interprétant à faire de ruinas et de casas deux attributs concurrents, más que ne portant de ce fait que sur le verbe attributif parecer. L'analyste, lui, pourra alors lire dans II une construction qui confère à más que une portée extraprédicative (dite "métalinguistique » dans le tableau 1), dans laquelle ce qui est mis en regard, c'est le degré de vérité d'un dire qui consiste à attribuer à une entité le nom de casas, et le degré de vérité d'un dire qui consisterait à lui attribuer le nom de ruinas. Le fonctionnement du dyptique más que diffère-t-il pour autant fondamentalement à l'instant de sa survenance? Manifestement non: l'acte d'interprétation, et l'analyse qui en découle, ne semble pas contradictoire avec le fonctionnement de más que en I, s'agissant d'une simple variation de portée de l'adverbe induite par l'après phrastique.

Ce que l'analyse en chronosyntaxe nous rappelle là, c'est que, case «comparaison » ou case « structure corrective » ou " additive », il y a bien là un seul et même signifiant más et un seul et même signifiant que, dont seule l'interaction avec la situation et l'entourage phrastique permet, en fin de parcours, de profiler une variation du sens de l'énoncé entier et de ranger la phrase entièrement constituée dans l'une des cases définies par l'analyste. Considérer cette variation en termes d'usurpation du signifiant, comme si celui-ci n'était qu'une forme d'habillement d'une catégorie abstraite se revêtant de lui pour se rendre visible, revient de ce point de vue à prendre les choses à l'envers. C'est accuser les gestes vocaux signifiants et les routines syntaxiques dans lesquels ils s'ancrent d'un «larcin » qui tient, en fait, à un simple acte d'interprétation par un interprétant en fonction de l'ensemble de la combinatoire syntaxique qui accompagne ces signifiants, et à une analyse a posteriori par un analyste placé en position surplombante d'obsevateur. Par ailleurs, en quoi l'interprétation I est-elle première par rapport à la II ? L'interprétant a-til besoin de passer par la I pour voir dans la II une déformation particulière de celle-ci ? Il semble bien plutôt que ces différentes catégories ne soient que différentes déclinaisons possibles, en fonction (ici) des dernières briques signifiantes portées à l'énoncé, d'un effet invariablement produit par les signifiants de et que à l'instant de leur survenance ; reste à définir quel il est et en quoi il diffère pour l'un et pour l'autre.

La démarche ici adoptée repartira de ces différents constats: celui qu'aucun déterminisme de l'entourage grammatical ne semble être recevable pour rendre compte du choix entre de et que, et que l'argument d'un évitement de la cacophonie ne l'est pas davantage ; celui que, s'agissant de deux signifiants différents, de et que ne pourront que provoquer deux effets différents à l'appréciation du récepteur et à leur survenance en tant que solveur d'une transitivité phrastique ouverte par más; et enfin, celui que más, de et que ne peuvent, dans le parcours d'un énoncé, provoquer que l'effet qui est le leur en tant que geste vocal et que l'on ne pourra les « accuser » de ce qui n'est pas en leur pouvoir. On tentera de capturer l'effet produit par más ... de ou más ... que à l'instant $t$ de leur survenance, en épurant l'analyse des catégories abstraites élaborées a posteriori par l'analyste, tout en veillant à définir une contribution de más ... de et más ... que compatible avec ces différentes catégories et permettant d'en rendre compte. Notre propos n'étant pas ici de dresser une typologie permettant d'étiqueter des structures mais de comprendre l'effet provoqué par la survenance de de ou de que en tant que solveur d'une transitivité phrastique ouverte par más, on n'excluera aucune construction répondant à ce simple critère de corrélation más que ou más $\sim$ de. Ce sont les cas où les deux 
signifiants sont possibles qui nous guideront en premier lieu, dans la mesure où ces cas où le locuteur a le choix sont sans aucun doute ceux qui révèlent le mieux ${ }^{21}$ l'effet produit sur la relation interlocutive par l'un par rapport à l'autre; les conclusions proposées pourront ensuite permettre d'éclairer les cas où seul que est (apparemment) possible.

\section{Que de : dissociation et association interlocutive}

\subsection{L'exemple de más que uno más de uno}

30 Commençons par comparer des cas tels que l'exemple (10) cité dans la partie précédente, où de et que alternent en position de répondant à más et en introduction d'un numéral, et pour lequel on avait noté des divergences possibles d'interprétation pour la variante avec que («estructura aditiva» ou «comparativa propia»). L'observation d'énoncés authentiques et en contexte, plutôt que d'exemples forgés par une grammaire, est particulièrement instructive en ce qui concerne le choix de que ou de de. Prenons les énoncés suivants dans leur contexte de réception étendu :

(13) [Sur le site Internet Babbel, destiné à l'apprentissage des langues étrangères, un billet de blog humoristique du webmaster avertit les apprenants des écarts possibles entre une langue apprise à l'école et les déformations habituelles de cette langue telles que pratiquées par les locuteurs natifs (verlan, troncations, etc.)] Francés hay más que uno. Quien llega al país galo como turista a veces tiene la sensación de estar pisando tierras movedizas verbales. El francés aprendido en la escuela se agota rápidamente en cuanto un francés empieza a hablar alegremente en argot o verlan.

(https://blog.babbel.com/es/frances-hay-mas-que-uno/, 17/01/2013, consulté le 8 avril 2018. C'est nous qui soulignons ${ }^{22}$

(14) [Le site du stade de football de Burgos, «El Plantío», annonce un match entre l'équipe burgalaise et celle de Santander. Le match sera crucial car il s'agit de la dernière chance pour l'équipe de Burgos de se qualifier pour la suite de la saison.] Llega a El Plantío uno de los partidos más esperados de la temporada, visita el coliseo blanquinegro el Racing de Santander, uno de los equipos fuertes del grupo. En juego más que tres puntos, un partido para recuperar la senda de la victoria, acercarse a los puestos de Copa y seguir luchando hasta el final por el playoff.

(http://www.burgoscf.es/ficha/Z460E3FC9-0BA9-02ED-D93B0C3D514E84EC/mas-

que-tres-puntos, consulté le 8 avril 2018. C'est nous qui soulignons) $)^{23}$

En (13), l'expression más que se positionne manifestement en réfutation d'une attente qui aurait pu être celle du lecteur. Avec le numéral uno, l'auteur situe son propre énoncé en écho à divers dictons espagnols à la forme négative, du type madre no hay más que una, padre no hay más que uno. L'auteur du blog, en adoptant la phrase Francés hay más que uno comme titre d'un billet destiné à illustrer les différences possibles entre un français appris en milieu scolaire et un français pratiqué par des locuteurs natifs de manière à prévenir les possibles surprises des voyageurs étrangers, se pose en tant qu'auteur du blog comme détenteur d'un savoir que les locuteurs ne soupçonnent pas; il surdistingue ainsi sa position de locuteur et semble faire une révélation : vous, lecteurs, pensiez avoir appris « le français » (seul et unique) ; il se trouve que, contrairement à tout ce que vous auriez pu espérer, francés hay más QUE uno.

Une telle interprétation en termes de réfutation et de surenchères par rapport à des attentes interlocutives coïncide alors avec une piste proposée par Sáez \& Sánchez López (2013: 70), selon qui un énoncé tel que (10b) avec que (se discutieron más que sesenta 
ponencias) ne serait viable que "como negación de una afirmación previa »; soit, en reconstruisant un contexte interocutif dans lequel un locuteur A aurait commenté $a$ posteriori le déroulement d'un colloque en affirmant que Se discutieron aproximadamente sesenta ponencias, l'allocutaire B se positionnant en faux contre cette affirmation et estimant que le colloque a été bien plus conséquent : no no, se discutieron más que sesenta ponencias $^{24}$. On pourra d'ailleurs remarquer que, dans ce dernier cas où il y a reprise corrective d'une phrase précédemment énoncée par l'allocutaire, la reconstruction d'un tel contexte amène presque naturellement à intensifier prosodiquement l'adverbe más, le détachant ainsi dans la chaîne parlée du signifiant voisin que. On ne pourra alors manquer de souligner la façon dont la décoalescence prosodique entre más, opérateur d'ajout accentué par B, et que sesenta ponencias, corrélat syntaxique de más qui reprend un point de repère précédemment énoncé par $A$, paraît remarquablement iconique de la dissociation interlocutive entre A et B qui résulte de l'ensemble ${ }^{25}$.

De manière tout à fait compatible avec cette analyse en termes de dissociation par rapport à des attentes ou des présupposés interlocutifs, mais déclinant cette valeur conflictuelle ou interlocutivement dissociative de que de manière légèrement différente, (14) présente un cas où que est lisible comme l'introducteur de « una coda 'aditiva de base no cuantitativa' » (Sáez \& Sánchez López 2013 : 71); entendons par là : l'idée qu'il s'agit de voir dans l'exemple (14) une référence à d'autres éléments en jeux à part ou en sus des trois points, plutôt qu'une référence à une quantité de points supérieure à trois ; cette interprétation se produisant dans «aquellos entornos donde más pueda adquirir por sí solo la interpretación 'más cosas' »"6 (Sáez \& Sánchez López 2013 : 72). Une telle lecture invite alors à percevoir l'objet comparé, plutôt que comme un ensemble fermé dont la constitution fait l'objet d'un consensus interlocutif, comme un ensemble ouvert pouvant inclure autre chose que les éléments déjà présents sur la scène interlocutive et que le locuteur présente comme étant présupposés par l'allocutaire.

Par contraste avec la structure en que dans un cas du type se discutieron más que sesenta ponencias, la structure en de (se discutieron más de sesenta ponencias) ne ferait que poser un repère numérique, sesenta ponencias apparaissant comme un point de référence interlocutivement partagé27 ${ }^{7}$ dans un énoncé tel que se discutieron más de sesenta ponencias, on pourrait imaginer que sesenta serait une sorte de "parangon", le nombre de communications à partir duquel un colloque apparaitt comme particulièrement imposant. La décoalescence prosodique remarquée dans le cas du même énoncé avec más ... que n'est plus de mise. C'est effectivement ce statut de simple repère que l'on reconnait dans les énoncés authentiques suivants, que l'on mettra aisément en contraste avec les (13) et (14) précédemment commentés :

(15) [Dans un livre de développement personnel, l'auteur compare les pensées intrusives censées empêcher les lecteurs de se livrer à leurs activités quotidiennes à des «dragons à sept têtes » et fournit des conseils sur la manière d'en venir à bout. L'un des chapitres est consacré aux cas où ces pensées intrusives concernent plusieurs problèmes différents]

Cuando hay más de uno. Algunos días tenemos que hacer varias cosas que no nos apetecen, hay más de un dragón que matar y no tenemos muy claro a cuál matar primero. La cosa es sencilla, escoge al más grande. Cuando mates al más grande, los demás te parecerán poco importantes o sencillos de matar.

(Alex Cumas, Cómo vencer al dragón de siete cabezas, consulté sur Google Books le 8 avril 2018) $)^{28}$

(16) [Consignes de mise aux normes d'un article par une revue scientifique]

Poner comas entre los apellidos y el nombre (o sus iniciales) del autor: Solamente se 
ponen comas para separar los autores (si hay más de uno). Las iniciales del nombre no llevan punto de no ser que sea el último autor, que lleva un punto para separarlo del título del libro.

(http://www.revistadyna.com/doc/como_citar_bibliografia.pdf, 13/12/2013, consulté le 8 avril 2018) ${ }^{29}$

Par contraste direct avec le más que uno précédemment commenté comme une réfutation d'attente dans l'exemple (13), le fait que le nombre d'auteurs d'un article à consigner en bibliographie puisse être supérieur à un - exemple (16) - ou que les préoccupations d'une personne puissent être supérieures à une - exemple (15) - est considéré comme une simple possibilité qui n'entre pas en contradiction avec une quelconque attente interlocutive. Au-delà de l'établissement de toute typologie grammaticale engonçant les énoncés dans des cases, se dessine alors une alternance de $\sim$ que, opposition entre deux formes qui s'apparient et s'opposent l'une à l'autre du point de vue du cadre réceptif qu'elles installent dans la relation entre locuteur et allocutaire. Que semble ainsi positionner más en réfutation d'une attente ou d'une projection supposée être celle de l'autre à qui on s'adresse; cette dissociation interlocutive se décline alors en effets de sens conflictuel - exemple (13) - ou additif - exemple (14) -, et d'autres que l'on détaillera plus avant. De, pour sa part, se positionne par contraste en introducteur d'un point de référence interlocutivement partagé.

\subsection{Más de que : une opposition à la croisée de l'interlocution, de la chronosyntaxe et de la cognématique}

Une telle proposition place l'alternance de / que dans le contexte de la comparaison d'inégalité en espagnol sur le terrain de la TRI (Théorie de la Relation Interlocutive) de Douay \& Roulland (2014), qui modélise les alternances grammaticales en fonction de leur effet sur le couple interlocutif (locuteur $\alpha$ - allocutaire $\beta)^{30}$, et propose de formuler les invariants des formes en termes de cadre réceptif:

Le cadre réceptif renvoie au type de relation configuré au niveau du système entre les pôles interlocutifs émetteur et récepteur, non encore instanciés à ce stade. Et c'est parce qu'il est configuré au niveau du système qu'il est parfaitement stable, donc invariant, non soumis aux aléas de l'usage [...]. Toute forme est révélatrice de la stratégie interlocutive mise en place par le locuteur pour installer une relation avec l'interlocuteur auquel il s'adresse. (Douay \& Roulland 2014 : 179-180)

Selon ce modèle, chaque forme signifiante, en plus de participer à la construction d'un sens énonciatif global, cristallise en retour une certaine configuration du couple interlocutif qui lui donne naissance ; ainsi, soit elle constitue ce couple comme la somme de deux entités distinctes, impliquant par là une surdistinction de la position locutive par rapport à l'interlocutive (configuration dite "de la distinction » ou "oppositionnelle ", appelée C1 - configuration 1), soit à l'inverse elle le constitue en une seule et même entité complexe sans distinction de la position locutive (configuration de la "continuité", appelée C2 - configuration 2) ${ }^{31}$. Les formes signifiantes se regroupent de ce fait en microsystèmes qui mettent en opposition des formes de type $\mathrm{C} 1$ et des formes de type $\mathrm{C} 2$, un exemple prototypique étant celui du micro-système de l'article en espagnol et en français : el (C2 présentant la notion comme connue de l'un et de l'autre, dans une continuité ne posant pas problème) un ( $\mathrm{C} 1$ présentant une notion non connue de l'interprétant ou qui déstabilise ses attentes). Dès lors que l'on cherche à comprendre l' effet des gestes signifiants dans la séquentialité de leur enchaînement, la formulation d'un 
invariant en termes d'effet interlocutif pour le geste vocal qu'est un signifiant parait tout particulièrement pertinent. Pour reprendre le cas de el et un, en chronosyntaxe, les articles avertissent l'interprétant de la survenance d'une notion (Bottineau 2014, Chevalier 199932) : el dit littéralement « une notion va être posée immédiatement après le présent signifiant ", ainsi el hombre un hombre; le choix de el posant, à l'instant de la survenance de l'article et avant même que la notion ne soit connue, que cette notion recouvrira un référent connu et identifié du locuteur comme de l'allocutaire, lesquels se trouvent alors associés dans cette relation à la notion; le choix de un, en revanche, pose à l'instant de sa survenance que la notion recouvrira un référent connu du locuteur mais encore impensé de l'allocutaire, l'un et l'autre se trouvant alors dissociés et le locuteur, surdistingué par rapport à l'allocutaire.

Pareillement, il semble que de et que, agissant en répondant de la transitivité phrastique ouverte par más, marquent la survenance d'un repère permettant de combler cette transitivité phrastique ; et que le choix de l'un ou de l'autre soit lié au cadre réceptif que le locuteur choisit d'installer. L'alternance de que formerait alors une paire dans laquelle que (forme de la dissociation interlocutive) installerait un cadre réceptif de type $\mathrm{C} 1$, et de (forme de l'association interlocutive) un cadre réceptif de type C2. La paire de que constitue par là une opposition transcatégorielle qui pourrait certes paraître choquante du point de vue d'une analyse distributionnelle classique, pour laquelle la reconnaissance d'une alternance préposition / conjonction ${ }^{33}$ ne va pas de soi; outre le fait que l'opposition semble pourtant fonctionner d'un point de vue interlocutif et chronosyntaxique, la proximité des deux opérateurs engagés dans cette alternance du point de vue du signifiant est tout à fait remarquable et plaide pour leur appariement l'un avec l'autre dans un micro-système d'opposition cohérent au moins là où le discours les met en concurrence. Ces opérateurs de et que - [de] et [ke] - sont liés par leur brièveté monosyllabique, par leur voyelle [e] commune qui semble être là en tant que résonnateur d'une consonne initiale qui se trouve être l'élément permettant d'opposer ces deux morphèmes l'un à l'autre : [d] et [k]. Pour les tenants d'une linguistique du signifiant dans laquelle la lecture de la forme même de ces gestes vocaux est pertinente pour saisir leur contribution à la construction du sens, une telle proximité entre les deux morphèmes, et leur mise en opposition par un seul élément qui se situe alors à un niveau submorphémique, est particulièrement remarquable. Les deux formants initiaux de de et que, les éléments de niveau submorphémique [d-] et [k-], se laissent alors décrire dans une linguistique du signifiant gestuel comme les deux cognèmes $\mathrm{T}$ et $\mathrm{K}^{34}$ :

Les cognèmes sont ces micro-signifiants élémentaires de niveau submorphémique qui, dans le cadre de réseaux d'oppositions au sein de systèmes grammaticaux, activent des micro-processus de synthèse du sens participant à la production de l'opérateur qui les intègre [...]. [Dans certains cas], la forme du geste interprétatif coïncide avec celle du geste articulatoire, faisant de la forme et du sens deux facettes analytiques du même geste. Chaque élément formateur est défini par un profil kinésique qui se relie diversement au contexte selon la situation considérée. (Bottineau 2016)

D'un point de vue articulatoire, $\mathrm{T}$ et $\mathrm{K}$ sont les symétriques l'un de l'autre :

On a analysé le formant $t$ comme la marque d'une occlusion interceptive terminale, en sortie de cavité orale. Si l'on se refère aux propositions de Toussaint ${ }^{35}$, son symétrique devrait être $k$ : occlusion interceptive initiale (vélaire), précoce dans la voie orale. (Bottineau 2010b : 33) 
Or, il se trouve que, en repartant de ce constat d'une opposition cognémique entre $\mathrm{T}$ et $\mathrm{K}$ on remarque la façon dont que ces deux cognèmes, en tant que gestes articulatoires, articulent l'interlocution : une interruption tardive du flux d'air pour T/D, pointant alors vers toi (allocutaire), face à une interruption précoce de $\mathrm{K}$, tirant le blocage dans la zone du moi (locuteur), permettent d'expliquer une répartition des opérateurs en fonction de leur mode de co-construction du sens dans la distribution intersubjective. Cette opposition a fait l'objet de nombreuses descriptions en espagnol dans le cadre d'une linguistique du signifiant ; ainsi, K caractérise-t-il des signifiants capables d'être investis en tant qu'opérateurs de subordination ou d'interrogation, dont des signifiants en $\mathrm{T}$ assurent le répondant : c'est le cas dans un micro-système exemplaire tel que cuanto tanto ou cual tal, mis au jour par Fortineau-Brémond (2012a : 143-153) et décrit comme suit par Bottineau (2012 : 47-48) :

Le marqueur cu- d'appréhension amémorielle de l'objet quantifié déclare le locuteur en état d'ignorance, soulève une question, et délègue à l'allocutaire la charge de compléter la connaissance qui fait défaut [...]. Le marqueur $t$ d'appréhension mémorielle, analogue à l'élément formateur du participe passé, fonctionne comme opérateur de saturation à valeur conclusive, et ne fait pas appel à l'allocutaire. [...] cuanto est une quantification subjective non stabilisée, non arrêtée par validation interlocutive, et à suspendre ou faire arrêter par l'allocutaire ; tanto et todo, des quantificateurs consensuellement ratifiés. (C'est nous qui soulignons)

C'est par cette même opposition cognémique, qui semble particulièrement à même de porter la distinction dissociation $\sim$ association interlocutive, que s'apparient ici les formes [ke] et [de] et en arrivent à former une opposition morphémique pertinente.

\subsection{Bilan}

Dès lors que l'on écoute les gestes signifiants en acte et considérés pour les effets qu'ils produisent sur les interprétants dans la séquentialité de leur enchaînement, ne dispose-ton pas là, avec l'interlocution, la chronosyntaxe et la cognématique, de trois outils méthodologiques s'épousant harmonieusement les uns les autres pour rendre raison de l'alternance entre l'appariement más que et l'appariement más de ? A partir de ces trois outils, reprenons l'étude d'un énoncé dans sa temporalité opératoire et en tenant compte de la bifurcation introduite par la survenance de de ou de que :

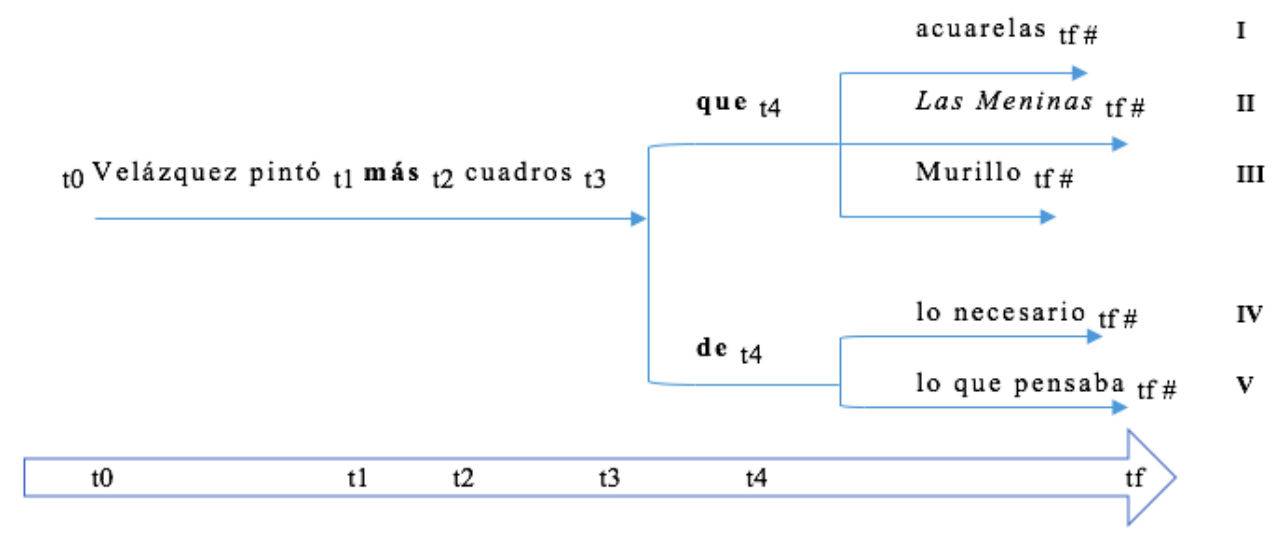

Figure 4.

En t2, más ouvre un espace de transitivité phrastique et proacte la survenance d'un repère. La survenance de ce repère est annoncée en $\mathrm{t} 3$ - $\mathrm{t} 4$ par la survenance de que ou de 
de. Si c'est que qui survient en $\mathrm{t} 3-\mathrm{t} 4$, l'interprétant en $\mathrm{t} 4 \mathrm{a}$ été averti de deux choses: premièrement, de la survenance immédiate du repère ; deuxièmement, que, du fait de son cognème $\mathrm{K}$ initial, marque que ce repère va marquer un écart par rapport à ses attendus ou, en tout cas, le locuteur le présente comme tel. Si c'est de qui survient en t3-t4, l'interprétant en $\mathrm{t} 4$ sait que le repère va être immédiatement énoncé, et de du fait de son cognème $\mathrm{T}$ initial l'avertit que ce repère ne sera qu'un point de référence considéré comme interlocutivement partagé et ne devant pas provoquer de dissensus interlocutif. Mais rien de plus; ce n'est que l'empan t4-tf qui permettra de déterminer dans quelle case de la typologie situer cet énoncé avec más que más de: comparativa correctiva (I), aditiva (II), propia (III), relativa (IV-V).

Une formulation de la contribution de de et de que dans ce type d'énoncés en termes de chronosyntaxe interlocutive semble en cela à même de rendre compte des différents effets de sens qui se déclinent dans le tableau 1. Reprenons à présent ces différentes cases tracées par l'analyste, qui ne sont sans doute qu'autant que déclinaisons différentes de cette systématique interlocutive.

\section{Déclinaisons de cette systématique interlocutive}

Reprenons à présent le tableau 1. Loin d'introduire des variations indues et scandaleuses de la "structure comparative", dès lors que la systématique interlocutive que configurent respectivement les dyptiques signifiants más que et más $\sim$ de a été définie, les structures autres que la dite "proprement comparative " paraissent couler de source: elles semblent n'être qu'une déclinaison possible de cette systématique interlocutive, qu'un résultat possible du cadre réceptif qu'installe chacun de ces deux dyptiques.

\subsection{Structures dites « additives » (más... que)}

L'énoncé (14) commenté précédemment aurait été rangé par un grammairien dans la case des « structures additives ». Cet effet additif de más ... que, on l'a vu, tient précisément à la dissociation interlocutive installé par que : le locuteur d'un tel énoncé, comme de l'énoncé (11) présenté en première partie de cette étude (Velázquez pintó más cuadros que Las Meninas, NGLE 45.4n), installe un ensemble ouvert de tableaux (cuadros) non restreints à ceux que le locuteur connait ou envisage ; le locuteur informe ainsi l'allocutaire d'un fait qui semble entrer en contradiction avec ses propres anticipations ou connaissances.

Les structures en no más que, dites " restrictivas » ou « excluyentes », que les grammaires pour apprenants de l'espagnol donnent pour équivalentes de solo ou de no ... sino, pourront être analysées comme le pendant négatif de ces structures dites « additives » :

(17) [Cinq ans après la guerre civile espagnole, sous la dictature franquiste. Ofelia vit seule avec sa mère Carmen, qui est enceinte de son nouveau mari Vidal, un capitaine de l'armée particulièrement malfaisant et qui traque sans relâche les maquisards anti-franquistes. Ofelia et Carmen partent rejoindre Vidal dans son campement. Dans la voiture qui les y conduit, Carmen demande à Ofelia d'appeler Vidal « père ». Ofelia est réticente.]

Es una palabra, Ofelia. No es más que una palabra.

(Del Toro, Guillermo [réalisateur]. El laberinto del fauno, 2006) ${ }^{36}$

En (17), avec no más que, la mère se positionne en réfutation des possibles protestations ou conceptions de sa fille; la valeur polémique, dissociative de más ... que lui permet de se 
placer dans une relation oppositive avec elle, contrecarrant ses éventuelles projections ( «le fait d'appeler cet inconnu 'père' lui donnera sur moi une autorité particulière, un droit d'affection que je ne veux lui autoriser »); d'où l'effet d'une restriction à la seule proposition du locuteur (« 'père' est un mot et ne signifie rien de plus »).

\subsection{Structures dites « correctives » (más... que)} palabra, toujours avec un effet interlocutivement dissociatif, déclare précisément qu'il faut voir dans le titre jsocorro! autre chose que ce à quoi le lecteur pourrait s'attendre ; dans ce texte écrit, Vázquez Montalbán se positionne donc par rapport à un alter imaginaire qui n'aurait pas perçu toute la portée politique de son titre :

(18) [Le 07/05/1988, soit treize ans après la mort de Franco, Manuel Vázquez Montalbán publie dans le journal El País une chronique intitulée ¡Socorro! qui fait état de dysfonctionnements politiques et juridiques dans une Espagne qui se veut pourtant démocratique. Il commente la manière dont s'est faite la Transition de la dictature franquiste à la démocratie, sans véritable rupture.]

¿Entienden ahora lo de la palabra ¡socorro!? Es algo más que una palabra, es una declaración de principios a la vista de que, casi diez años después de la entronización democrática, el pacto que permitió la Transición revela cuánta miseria hubo que tragarse, cuánto fascismo latente, del viejo y del nuevo, metido en los aparatos de Estado y en la moralidad gobernante. ${ }^{37}$

On retrouve alors une structure dite "corrective». L'effet dissociatif de C1 est probablement tout particulièrement évident dans le cas de ces structures, dont l'effet « correctif » entre en pleine cohérence avec le caractère dissociatif de que dans la relation interlocutive. Ainsi, un énoncé fréquemment utilisé par les grammaires tel que le suivant:

(19) Es trabajador más que inteligente..$^{38}$

51 doit être compris dans le cadre d'un échange interlocutif ou d'une théorie de l'esprit dans laquelle tu attribue les bons résultats du délocuté à son intelligence, et où je corrige, rectifie en les attribuant bien plutôt à son travail : la construction est alors conflictuelle et polémique du point de vue interlocutif, elle met en débat les positions du locuteur et de l'allocutaire.

Les publicitaires font grand usage de ces structures, qui leur permettent de donner aux lecteurs la sensation que le produit proposé ouvre des perspectives insoupçonnées. Il permet d'appréhender la notion introduite (le produit) non pas de manière continue et sans heurt explicite pour l'allocutaire, mais au contraire de façon discontinue par rapport aux projections habituelles de celui-ci, lui ouvrant par là un espace à découvrir (volontiers présenté comme mystérieux ou onirique) qui sur-distingue, valorise la présentation qu'en fait le locuteur par rapport à une présentation concurrente plus traditionnelle :

(20) Chivas, más que un whisky... es una leyenda.

(Google.es, http://www.revistasexcelencias.com/excelencias-gourmet/gourmet-elperfecto-equilibrio/publireportaje/chivas-mas-que-un-whisky...-es-una-l, consulté le 8 juin 2018)

(21) Los Miserables. Más que un musical, una leyenda.

(Google.es, http://ciudaddecultura.org/plazas-y-patios-escenicos/details/721.html, consulté le 8 juin 2018)

(22) Más que una navaja, una leyenda. Victorinox, es un un sello que honra la 
cultura y los valores de Suiza.

(Google.es, https://h-ejecutivo.com/mas-que-una-navaja-una-leyenda/, consulté le 8 juin 2018 $)^{39}$

\subsection{Más de lo ... / más que lo ... + adj. ou participe, más de la cuenta}

Les repères en lo + adjectif ou participe peuvent, eux aussi, être introduits par de comme par que, ce qui crée deux cadres réceptifs opposés : « más de lo habitual » non conflictuel - comme en (4) où le temps requis pour rentrer de México est présenté comme faisant consensus interlocutif -, "más que lo habitual» conflictuel - comme en (5) où, en déclarant que leur émission radio a été « improvisée », les présentateurs précisent «más que lo habitual » comme pour anticiper une réponse de leurs auditeurs habitués à l'improvisation dans leurs programmes: más que lo habitual vient ici sur-distinguer le jugement d'improvisation du locuteur par rapport au degré auquel les interlocuteurs sont habitués.

(4) María Elena pasa otras dos semanas sin salir, sin peinarse, sin lavarse. Cree que se va a morir. Y está conforme. Higinio tarda más de lo habitual en volver de la ciudad de México, pero vuelve con un niño.

(CORPES XXI, José Montelongo, Quincalla, México 2004. Cité en introduction)

(5) [Sur une plateforme Internet, une radio propose de réécouter les podcasts de ses anciennes émissions. Un court texte de présentation commente, à propos de l'une de cellesci:]

En este programa improvisado (aún más que lo habitual) damos unas cuántas recomendaciones para ver en Netflix, también hablamos un poco sobre el manga de Dr. Stone.

(Google, https://itunes.apple.com/us/podcast/mal-vivir/id1219873916?mt=2 , consulté le 8 juin 2018. Cité en introduction)

$\mathrm{Si}$, par un adjectif ou participe substantivé qui se place juste après de lo, le locuteur précise les conditions d'accord interlocutif d'un point de référence partagé (lo normal, lo convenido, lo esperado, lo acordado, lo prometido, lo exigido, lo autorizado, lo programado...), une expression fort courante en espagnol contemporain permet de neutraliser les conditions de cet accord et de simplement lexicaliser (au sens premier du terme : réaliser une idée par un terme du lexique qui permet de la rendre présente sur la scène verbale), sans plus de précisions, le repère qui fait consensus interlocutif : más de la cuenta. Ainsi, dans l'échange suivant :

(23) [Miguel, le personnage principal du film, vient d'être quitté par sa femme et s'occupe seul de sa belle-mère malade. Dans cette séquence, il discute devant chez lui avec Yasmina, une jeune femme qu'il a récemment rencontrée; sa belle-mère fait un malaise et Yasmina aide alors Miguel à la monter dans l'appartement. Yasmina se met à interroger Miguel sur sa situation.]

Yasmina: Es muy guapa su madre.

Miguel: No, no es mi madre.

Y: ¿Una tía?

M: No, es mi suegra.

Y: Ah, que es usted casado. Claro, que como no lleva usted anillo... Aunque esas cosas ya no se llevan. [...] Y ¿la hija? O sea, ¿la mujer de usted? ¡Uy perdón! Ya estoy preguntando más de la cuenta. Es un defecto que tengo. La falta de costumbre.

(Albaladejo, Miguel [réalisateur]. El cielo abierto, 2000) ${ }^{40}$

Yasmina, face à l'embarras de Miguel et sur un ton déterminé qui ne laisse aucune prise à Miguel pour la détromper courtoisement, constate pour son interlocuteur comme pour elle (alors fédérés par cet accord interlocutif) que ses questions dépassent un stade 
consensuellement ratifié comme ne relevant plus de la politesse. Cuenta (du verbe contar, compter) permet de désigner l'atteinte de ce stade sans préciser quel il est ni comment il a été ratifié. L'expression abolit tout différentiel interlocutif et au contraire, réunit les deux personnages dans un accord interlocutif tacite (souligné par l'utilisation par Yasmina de más $D E$ la cuenta) autour du dépassement d'une borne convenue. On remarquera qu'une recherche Google (consulté le 11/03/2018) en expression exacte donne 2,5 millions de «más de la cuenta » et seulement 5 résultats pour «más que la cuenta ", parmi lesquels :

(24) [L'article discute de la possibilité, pour un serveur, de faire payer un supplément au client pour lui avoir apporté l'addition ou le terminal de paiement à sa table] En cuanto a la necesidad de volver a la mesa con un datáfono, es posible que se acabe aplicando una tarifa disuasoria, ya que 'el datáfono pesa mucho más que la cuenta, está más lejos y utilizarlo requiere de una formación', aseguran los camareros.

http://www.elmundotoday.com/2017/10/los-camareros-cobraran-un-suplementopor-traer-la-cuenta/ (consulté le 11 mars 2018. C'est nous qui soulignons) ${ }^{41}$

Pareillement, « comer más que la cuenta » renvoie dans ces cas immédiatement au fait de manger davantage que ce qui est payé selon la note. Dans más de la cuenta, il s'agit donc bien d'un simple repère correspondant à une quantité ou à un "stade » atteint et qui fait consensus; l'usage de que y serait alors contradictoire et on ne le trouve bien que dans des cas où "la cuenta" renvoie à tout autre chose qu'un point de repère interlocutivement partagé non précisé.

\subsection{Et les « comparativas propias » ? (más... que)}

57 Jusqu'ici, les différentes cases du tableau 1 déclinent parfaitement l'opposition de de et que en tant que répondants à más et posent un repère, le premier annonçant ce repère comme simple point de référence consensuellement ratifié, le deuxième l'annonçant comme objet de dissensus interlocutif. On pourrait à présent s'étonner de ce que, dans les dites comparativas propias du type Alberto es más alto que Juan mettant en parallèle deux entités au regard d'un paramètre et où un interprétant ne détecterait aucune nuance corrective ni additive, seul que puisse apparemment apparaître au détriment de *Alberto es más alto de Juan. L'espagnol semblerait-il par là marquer systématiquement par une forme que typique du dissensus interlocutif ce que les grammairiens identifient comme les comparativas propias? Et pourquoi le ferait-il?

La question appelle plusieurs types de commentaires. En premier lieu, un regard sur la diachronie nous apprend qu'une phrase du type *Alberto es más alto de Juan aurait été tout aussi viable que Alberto es más alto que Juan en espagnol médiéval et classique. Romero Cambrón (1995 et sq.) explique que de y était choisi lorsque le comparant (ou deuxième terme de la comparaison) correspondait à une forme de parangon socialement défini et dont il était de ce fait possible pour les interprétants d'extraire la caractéristique servant de paramètre de la comparaison :

[...] el 2T [segundo término de la comparación] actúa como un estándar al que pragmáticamente se le atribuye la posesión (generalmente, en un alto grado) de la propiedad mensurada. [...] es el saber pragmático el que permite reconstruir ese otro grado de la propiedad que funciona en la confrontación como segundo constituyente comparado [...]. [El 2T] representa un modelo a partir del cual es posible determinar, a través de nuestro saber pragmático, un segundo grado de la propiedad contrastada. (Rombero Cambrón 1997 : 342-343) ${ }^{42}$ 
59

exemple forgé en espagnol contemporain, elle explique que l'espagnol ancien aurait choisi de dans *Juan tiene alumnos más traviesos de Zipi y Zape ${ }^{43}$ où Zipi et Zape servent de parangons de la malice enfantine - mais, sans doute, que dans une phrase où le comparant est tout autre chose qu'un terme socialement reconnu comme parangon; ainsi, Juan tiene alumnos más traviesos que tus sobrinos. N'est-on pas fondé à reconnaître là une répartition le fonctionnement précédemment commenté de de et que en tant qu'opérateurs d'association interlocutive (dans la confrontation à un parangon reconnu comme partagé) et de dissociation interlocutive (lorsque ce n'est pas le cas) ? C'est effectivement ce que l'on trouve dans les exemples 25 à 27 - où, vérification faite dans les transcriptions modernisées ajoutées sous chaque exemple, que est attendu en espagnol contemporain. Un mal qui ne peut être soulagé que par la mort (25), Judas (26) et le soleil (27) sont respectivement pris comme les parangons des maux humains, des traîtres et des sources de clarté, le locuteur présentant cette caractéristique comme faisant consensus :

(25) ¿Qué mayor desdicha puede ser - replicó Sancho Panza - de aquella que aguarda al tiempo que la consuma y a la muerte que la acabe ?

(Cervantes, Quijote I, XV, 193. Cité par Romero Cambrón 1998 : 121)

Transcription modernisée: Pues, ¿qué mayor desdicha puede ser que aquella que aguarda al tiempo que la consuma y a la muerte que la acabe? (éd. Andrés Trapiello. Barcelona, Planeta 2015 : 132)

(26) Don renegado malo. de Judas muy peor / no sé por ti qui quiera rogar al Criador.

(Berceo, Milagros, vv. 824cd, cité par Romero Cambrón 1995 : 159)

Transcription modernisée: Don renegado malo, que Judas mucho peor, / no sé por ti quién quiera rogar al Criador. (éd. Daniel Devoto. Madrid, Castalia 1969 : 131)

(27) Vida que non da fin e salud perdurable. claridat más de sol. firme paz e estable, ligerez más de viento, sotileza mirable, tal regno de tal rey es mucho deseable.

(Berceo. Loores, est. 192. Cité par Romero Cambrón 1995 : 175)

Transcription modernisée : [Vida que da] más claridad que el sol, más ligereza que

el viento [...], tal reino de tal rey es muy deseable. (Romero Cambrón $1998: 63)^{44}$

Dans ces différents exemples, ce que disent les transcriptions modernisées, c'est que l'espagnol contemporain - par rapport à un espagnol médiéval qui laissait le choix aux locuteurs - semble avoir incrusté, dans la routine énonciative par laquelle sont confrontés deux éléments au regard d'un paramètre, la distanciation relative du locuteur par rapport au standard.

Deux remarques à ce sujet. Premièrement, remarquons que dès lors que l'on prend en compte non pas seulement une structure syntaxique décontextualisée et désincarnée, mais l'expérience vécue des locuteurs dans laquelle une comparaison se fait à l'adresse d'un interprétant (réflexif ou interpellé), cette incrustation semble relativement naturelle. En effet, si on considère que :

[...] comparer, ce n'est pas déclarer une proposition vraie et l'autre fausse, mais les hiérarchiser: le locuteur opte en faveur de l'une au détriment de l'autre. [...] la proposition contenue dans le standard, renvoyée pour la forme à un énonciateur distancié par que, n'est pas celle que le locuteur choisit de valider préférentiellement. (Bottineau à paraître)

alors, adoper une forme que révélant le « [...] désaccord relatif du locuteur par rapport à la proposition non sélectionnée [...] sans pour autant la considérer comme intrinsèquement non valide » (Bottineau à paraître) ne paraît pas contradictoire.

Pour autant, cela signifie-t-il qu'un locuteur espagnol d'aujourd'hui est entièrement contraint et n'a en aucune manière, dans une mise en parallèle du type Alberto es más alto 
que Juan entre deux entités au regard d'un paramètre, la possibilité de marquer que le deuxième terme n'est qu'un simple point de référence interlocutivement partagé, et de neutraliser par là le différentiel interlocutif ? Considérons les cas (28) à (30).

(28) Este periodo también se conoce como Periodo Medieval Cálido, y fue seguido entre 1450 y 1890 por la llamada Pequeña Edad de Hielo - o Pequeña Era Glacial -, en la que la temperatura promedio global fue de medio a un grado centígrado más baja de lo que es hoy en día. Varias explicaciones se han propuesto para estas oscilaciones climáticas a escala corta de tiempo.

(CORPES XXI. Schifter, Isaac; González-Macías, Carmen: La Tierra tiene fiebre. México D. F.: FCE, SEP, CONACYT, CAB, 2005)

(29) SILVIA.- Pues dile a Clarisa que se olvide de la "mujer" que le han enseñado a ser y de la que han querido que sea. ¡Qué deje de hacerse la víctima, por favor!

PILAR.- De seguir tu consejo acabará más sola de lo que está ahora...

SILVIA.- Parece mentira que todo se resuma a un como no acabar solas.

(CORPES XXI. Marquet, Toni: Ausencias. Alicante: Biblioteca Virtual Miguel de Cervantes, 2002)

(30) Sí que es cierto que el vino es bastante más complejo de lo que es la cerveza por lo que nuestros amigos los cerveceros lo tuvieron mucho más sencillo de conseguir, creo que hay cervezas sin alcohol que yo no pondría la mano en el fuego si tiene o no alcohol.

(CORPES XXI. Orio, Pablo: «Vino sin alcohol. II parte», www.puntovino.es, 2011-07-21) $)^{45}$

Dans chacun de ces cas, est en jeu une mise en parallèle entre deux entités - la température de la " petite ère glacière » et celle d'aujourd'hui, en (28) - au regard d'un paramètre - basse. On aurait pu attendre [...] fue más baja que hoy en día (28), tout comme más sola que ahora en (29) et más complejo que la cerveza en (30). Mais l'utilisation de de permet de présenter la période d'aujourd'hui (hoy en día) comme le point de référence partagé entre scripteurs et lecteurs. Ainsi, dans une phrase du type Alberto es más alto que Juan, le locuteur marque sa distanciation; en revanche, dans une phrase du type Alberto es más alto de lo que es Juan, la grandeur de Juan est considérée comme un parangon interlocutivement ratifié par rapport auquel il est possible de mesurer, par contraste, d'autres grandeurs. Certes, cette possibilité pourra être jugée relativement " lourde » : de fait, elle implique un effort supplémentaire de la part du locuteur pour neutraliser, inhiber ce qui est dorénavant inscrit dans la structure.

\section{Conclusion}

Les deux marqueurs du standard de et que entrant en répondant avec más en espagnol produisent une série d'effets bien différenciés dont il est possible de rendre compte de manière unifiée à partir de la configuration de la relation interlocutive qu'installe le choix de l'un ou de l'autre. La contribution de chacun de ces brefs signifiants à la dynamique de construction du sens se réduit alors à un apport opératoire épuré, dépouillé, qui se limite au simple geste articulatoire considéré en chronosyntaxe et dans la relation locuteur allocutaire : corrélé à más, que, marqué par un geste [k] d'interception précoce analogue à l'élément formateur des signifiants de l'interrogation, installe une forme de dissociation interlocutive lui permettant dans certains contextes de prendre des effets correctifs, contrastifs, restrictifs ou additifs; de, au contraire, avec son geste d'interception tardive et englobant, installe une forme d'association entre les deux interlocuteurs autour d'un standard érigé en point de référence consensuellement ratifié 
et interlocutivement partagé. Dans ce cadre, les structures habituellement dites " additives", " correctives ", « comparatives relatives", « expressions numérales complexes » et autres, n'apparaissent plus comme des déformations indues, difficiles à classer et presque monstrueuses d'une structure de "comparaison propre». Elles apparaissent au contraire, et au même titre que cette dernière, comme autant de déclinaisons possibles de l'effet invariant produit par más de d'un côté ou de celui produit par más que de l'autre, dont il devient possible de rendre compte sans avoir à les dériver d'une structure syntaxique originale et parfaite qu'ils déformeraient ou dont ils usurperaient la forme.

On a donc proposé ici, attaché à más de et más que, un effet inhérent au signifiant en tant que geste matériel permettant de rendre compte de la multiplicité deur leurs usages à l'interprétation. Le cadre réceptif invariant proposé a permis de proposer une unité cohérente fondant la diversité des emplois, et d'introduire un principe de causalité liant forme et sens et permettant de réaliser des prédictions sémantiques par l'observation des signifiants dans leur matérialité. Toutefois, on s'est concentrée ici sur la contribution de de et de que considérés dans leur alternance au sein d'une routine syntaxique qui les place en concurrents l'un de l'autre. Cela signifie-t-il que la contribution ici proposée pour ces signifiants est la même dans d'autres contextes, lorsque la reconnaissance d'une opposition et d'un micro-système signifiant [de] [ke] n'est pas pertinente ? La question est légitimement ouverte et la réponse, loin de s'imposer comme une évidence. D'un côté, il est certain que poser une variation possible en dehors de cet appariement [de] [ke] pourrait apparaitre comme une contradiction relative face à notre recherche d'invariant. D'un autre côté, une approche comme la nôtre, qui suppose de ne pas faire abstraction d'un système de pratiques pour expliquer les propriétés d'un système linguistique, n'estelle pas cohérente avec l'idée d'être ouvert à la possibilité que l'effet d'un signifiant puisse être soumis à un profilage vicariant en fonction de son inscription dans un réseau de signifiants qui tour à tour pourront venir s'apparier à lui (ainsi que vs. de ici, mais aussi de vs. a ailleurs au sein même du micro-système des prépositions) ? Dans ce cas, la répartition dissociation $\sim$ association interlocutive pourrait-elle différer selon les appariements qui se font et se défont aléatoirement au gré du discours, des analogies et des oppositions, des alternances perçues par les locuteurs? En dernier lieu, la réduction de l'effet du signifiant à un simple geste ou coordination de gestes sous-tendant le profilage d'un cadre réceptif en situation interlocutive, plutôt que la définition d'un invariant de type représentationnel ou schématique si abstrait soit-il, pourrait-il être à même de concilier l'unité d'un geste signifiant et la possibilité de profilages distincts selon ses inscriptions réticulaires? On ne tranchera pas ici et ces questions restent ouvertes.

L'un des enjeux actuels d'une linguistique de l'expérience, visant à l'humanisation des systèmes linguistiques appréhendés phénoménologiquement en actes via la parole comme conduite de production du sens, est précisément la mise en cohérence des différents modèles ici mobilisés, représentatifs d'une linguistique incarnée par le signifiant considéré pour ses effets perceptuels (cognématique et submorphémies), processuelle dans la considération de l'émergence dynamique du sens (chronosyntaxes), située dans les interactions verbales et distribuée en ce que le sens est conçu comme un co-construit plus qu'un préconstruit encodé par un centre cognitif locutif seul (relation interlocutive). Cette étude se veut un jalon dans cette direction, sur un terrain que nous avons perçu comme particulièrement propice au croisement de ces modèles théoriques ; le croisement 
et la mise en dialogue mérite d'être consolidée à la lumière d'autres faits linguistiques, dans diverses langues.

En ce qui concerne la comparaison, une telle approche suppose de concevoir la langue non pas comme un appareil formel et abstrait conçu pour un locuteur se dressant seul face à un monde qu'il commenterait, comparant pour lui-même les éléments qu'il y voit, mais comme un ensemble cohérent de techniques gestuelles émergeant de la co-action de locuteurs et allocutaires cherchant sans cesse à se coordonner les uns avec les autres. Les signifiants, ainsi que les routines syntaxiques dans lesquels ils sont ancrés, constituent autant d'interfaces permettant cette coordination interpersonnelle; leur lecture, dans leur matérialité gestuelle saisie à la croisée de la chronosyntaxe et de l'interlocution, permet de comprendre la manière dont se configurent ces coordinations dans une langue particulière. La mise en contraste de l'alternance des signifiants más de más que en espagnol avec les signifiants d'autres langues (français plus de $\sim$ plus que, portugais mais que mais do que, etc.), au-delà du constat de l'existence d'une alternance récurrente, serait probablement à même de saisir les différences dans la façon dont chaque langue configure la relation interlocutive et décline les configurations possibles de cette relation dans l'expression de la comparaison.

\section{BIBLIOGRAPHY}

ALLEN J.J. (1958). « Notes on the use of de and que with antes and después », Hispania 41, 504-510.

ANDER MENDIA, Jon. (à paraître). « One more comparative : the de / que alternation in Spanish comparatives », Natural Language \& Linguistic Theories, en ligne http://people.umass.edu/ jmendiaaldam/JA-files/mendia-1MoreComp.pdf.

AUER, Peter (2009). « On-line syntax : thoughts on the temporality of spoken language », Language Sciences 31, 1-13.

BEDEL, Jean-Marc. (1997). Grammaire de l'espagnol moderne. Paris : PUF.

BELLO, Andrés. (1847). Gramática de la lengua castellana destinada al uso de los americanos, edición crítica y estudio preliminar de R. Trujillo, Santa Cruz de Tenerife, Instituto de Lingüística Andrés Bello / Aula de Cultura del Cabildo Insular de Tenerife.

BOLINGER D. (1950). « The comparison of inequality in Spanish », Language 26, 28-62.

-, (1953). « Addenda to 'The comparison of inequality in Spanish' », Language 29, 62-66.

BOTTINEAU, Didier. (2000). « Son, sens et traduction : de l'insignifiance au réinvestissement grammaticalisé de i et a en anglais. Etude de quelques marqueurs appartenant au syntagme nominal et conséquences traductologiques », M. Ballard (éd), Oralité et traduction (p. 43-77). Arras : Artois Presse Université.

-, (2010a). «L'émergence du sens par l'acte de langage, de la syntaxe au submorphème », M. Banniard \& D. Philps (éds), La fabrique du signe, Linguistique de l'émergence (p. 299-325). Toulouse : Presses Universitaires du Mirail. 
-, (2010b). « La submorphologie grammaticale en espagnol et la théorie des cognèmes ». Dans G. Le Tallec-Lloret (dir.) Vues et contrevues. Actes du XII ${ }^{\mathrm{e}}$ Colloque international de Linguistique ibéro-romane, Université de Haute Bretagne - Rennes 2 (p. 19-40). Limoges : Lambert Lucas.

-, (2011). « Parole, corporéité, individu et société : l'embodiment entre le représentationnalisme et la cognition incarnée, distribuée, biosémiotique et enactive dans les linguistiques cognitives », Intellectica 56, 187-220.

-, (2012). « Submorphologie et processus aspectuels en morphologie grammaticale espagnole », dans G. Luquet (éd.), Morphosytaxe et sémantique espagnoles. Théorie et applications (p. 37-56). Paris : Presses Sorbonne Nouvelle.

-, (2014). « Grammaire enactive et didactique du FLE », dans C. Martinot et A. Pégaz Paquet (éds.), Innovations didactiques en français langue étrangère (p. 185-200). CRL : Paris.

-, (2016). «Linguistique incarnée et « énactivisme » : quelles collaborations possibles avec les neurosciences ? » Dans A. Rabatel, M. Temmar \& J.M. Leblanc (éds.), Sciences du langage et neurosciences. Actes du colloque ASL 2015 (p. 211-232). Limoges : Lambert-Lucas.

-, (2017a). « Langagement (languaging), langage et énaction, a tale of two schools of scholars : un dialogue entre biologie et linguistique en construction », Signifiances 1/1, 11-38.

-, (2017b). « Du languaging au sens linguistique ». Intellectica 68, 19-67.

-, (à paraître). « La négation du « standard » de la comparaison : une approche instructionnelle ». BRUCART J. M. (2003). « Adición, sustracción y comparación : un análisis composicional de las construcciones aditivo-sustractivas del español », en F. Sánchez Miret (ed.), Actas del XXIII Congreso Internacional de Lingüística y Filología Románica (p. 11-60), Tubinga : Max Niemeyer. CHEVALIER, Jean-Claude (1999). « Chrono-syntaxe et collocation des pronoms compléments en espagnol ». Dans Mélanges de linguistique, sémiotique et narratologie dédiés à la mémoirede Kasimir Mantchev, à l'occasion de son $60^{\mathrm{e}}$ anniversaire (p. 68-90). Sofia : Éditions Colibri.

CHEVALIER, Jean-Claude, MOLHO, Maurice \& LAUNAY, Michel (1984). « La raison du signifiant », Modèles linguistiques, VI, 2, 1984, p. 27-41.

-, « Pour une linguistique du signifiant », Cahiers du CRIAR 6, 1986a, 95-99.

- (éd.) (1986b), Le signifiant, numéro de la revue Langages.

COL, Gilles. (2017). Construction du sens : un modèle instructionnel pour la sémantique. Bern : Peter Lang.

COL, Gilles, APTEKMAN, Jeanne, GIRAULT, Stéphanie, VICTORRI, Bernard. (2010). « Compositionnalité gestaltiste et construction du sens par instructions dynamiques ", CogniTextes 5. URL : http:// journals.openedition.org/cognitextes/372

DELPORT Marie-France. (2004). Deux verbes espagnols : aver et tener, étude lexico-syntaxique. Perspective comparative, Paris : Éditions Hispaniques.

DOUAY Catherine \& RoulLAND Daniel. (2014). Théorie de la relation interlocutive. Sens, signe, réplication, Limoges : Lambert-Lucas.

FUCHS, Catherine. (2014). La comparaison et son expression en français. Paris : Ophrys.

FORTINEAU-BRÉMOND, Chrystelle. (2012a). La corrélation en espagnol contemporain. Morphologie, syntaxe et sémantique. Rennes : Presses Universitaires de Rennes. 
-, (2012b) « La structure corrélative más... más... : du signifiant à la syntaxe », dans Luquet G. (éd.), Morphosyntaxe et sémantique espagnoles. Théories et applications (p. 73-90), Paris : Presses Sorbonne Nouvelle.

-, (à paraître). « La structure corrélative más... más... et ses 'variantes' : submorphémie, syntaxe et sémantique ", dans É. Blestel \& C. Fortineau-Brémond (éd.), Le signifiant sens dessus dessous. Submorphémie et chrono-analyse en linguistique hispanique. Limoges : Lambert-Lucas.

GERBOIN, Pierre \& LEROY, Christine. (1991). Grammaire d'usage de l'espagnol contemporain. Paris : Hachette Éducation.

GOODY, Jack (1996). L'homme, l'écriture et la mort. Paris : Les Belles Lettres.

GUARDIA NIETO, P. (1990). «Alternancias de/que en las comparativas de desigualdad », en C. Martín Vide (ed.), Actas del V Congreso de Lenguajes Naturales y Lenguajes Formales : 575-584.

GUTIÉRREZ ordóñEZ S. (1992). « Más de - más que », Contextos X / 19-20, 47-86.

-, (1994a). Estructuras comparativas, Madrid : Arco Libros.

-, (1994b). Estructuras pseudocomparativas, Madrid : Arco Libros.

LÓPEZ GARCÍA, Ángel. (1983). « La comparación en español : estructura fraseológica y estructura oracional », en Serta Philologica F. Lázaro Carreter, I, 315-327.

MACCHI, Yves. (2000). «L'anticipation syntaxique de l'attribut. Essai de chronosyntaxe ». Dans A. Résano (éd.), Linguistique hispanique, Actes du VIIIème Colloque de Linguistique Hispanique (p. 395-413), Nantes : CRINI.

-, (2006). «Transitivité et intransitivité : propriétés du mot ou effets du processus phrastique ? Chronosyntaxe (VI) ». dans G. Luquet (éd.), Le signifié de langue en espagnol (p. 115-134). Paris : Presses Sorbonne Nouvelle.

-, (2008). « On vous le ramènera, votre mari ! : Esquisse d'une topologie du signifié. Chronosyntaxe (III) », Chréode - Vers une linguistique du signifiant, revue de linguistique hispanique et romane, $\mathrm{n}^{\circ} 1$, Paris IV : Éditions Hispaniques, 141-178.

-, (à paraître). « Du sens et de la signifiance du substantif monosyllabique espagnol pie Chronosémantique (I) », communication à la Journée d'études La linguistique du signifiant, Approches et domaines d'application, 6 juin 2014, Org. José Vicente Lozano, Université de Rouen. A paraître dans les Cahiers de l'ERIAC.

MELLo G. de. (1977). «Que and de as translations of than », Hispania 60 : 510-511.

NGLE et NGLE Manual : voir Real Academia Española \& Asociación de Academias de la lengua Española.

ORTEGA SANTOS, Iván. (2013). « Microvariation in Spanish Comparatives », Catalan Journal of Linguistics, 12, 175-192.

PLANN S. (1984). « The syntax and semantics of más/menos ... que versus más/menos de in comparatives of inequality », Hispanic Linguistics 1, 191-213.

PRYTZ, Otto. (1979). «Construcciones comparativas en español », Revue Romane, 14/2, 260-278.

REAL ACADEMIA ESPAÑOLA \& ASOCIACIÓN DE ACADEMIAS DE LA LENGUA ESPAÑOLA. (2009). Nueva gramática de la lengua española, (T.1 : Morfología. Sintaxis I; T. 2 : Sintaxis II), Madrid, Espasa Libros. [NGLE].

-, (2010). Nueva gramática de la lengua española. Manual. Madrid, Espasa Libros. [NGLE Manual]. 
ROJAS NIETO, Cecilia. (1990). «El término de las construcciones comparativas de desigualdad : de o que », en V. Demonte \& B. Garza Cuarón (éd.), Estudios de lingüística de España y México (p. 225-241), México : UNAM.

ROMERO CAMBRÓN, Ángeles. (1995). « La construcción comparativa preposicional en castellano antiguo ». Revue de linguistique romane 59, 159-190.

-, (1997). « De nuevo sobre la alternancia que/de en el segundo término comparativo ». Verba 24, 335-348.

-, (1998). Historia sintáctica de las construcciones comparativas de desigualdad. Cuenca: Ediciones de la Universidad de Castilla-La Mancha.

ROULLAND, Daniel. (2017). « Langage et réplication ». Intellectica 68, 69-97.

SÁEZ Luis \& SÁNCHEZ LóPEZ Cristina (éd.). (2013). Las construcciones comparativas, Madrid : Visor Libros.

SÁEZ DEL ÁlAMO, Luis. (1999). « Los cuantificadores : las construcciones comparativas y

superlativas ", dans I. Bosque \& V. Demonte (éd.), Gramática descriptiva de la lengua española, vol. 1. (p. 1129-1188), Madrid : Espasa Calpe.

SÁENZ H. (1940). « The Spanish translation of than », Hispania 23 : 326-330.

SICOT DOMínGUEZ Marisol (2010a). « Le signe /mas/ : entre la quantification et l'adversation », dans Luquet G. (éd.), Morphologie et syntaxe de l'espagnol. Méthodes d'approche (p. 111-123), Paris : Presses Sorbonne Nouvelle.

-, (2010b). « Le signe /mas/: du signifié au signifiant », dans G. Le Tallec-Lloret (éd.), Vues et contrevues. Actes du XIIe colloque international de linguistique ibéro-romane, Université de HauteBretagne, Rennes 2 (p. 305-312). Limoges : Lambert-Lucas.

SOLÉ Y. (1982). « On más/menos ... que versus más/menos ... de comparatives », Hispania 65 : 614-619.

SPAULDing R. R. (1962). « De l(, la, lo, los, las) que vs. que (el, la, lo, los, las) que or the force of tradition », Hispania 45/2, 309-314.

TOUSSAINT, Maurice. (1983). Contre l'arbitraire du signe. Paris : Didier Érudition.

\section{NOTES}

1. «*Charles a bu plus de trois bières qu'Alfred ». (notre traduction)

2. L'approche chronosyntaxique part du principe qu'une phrase apparaît aux interprétants comme un "défilé successif [...] d'unités signifiantes " (Macchi 2006 : 115) dont les effets sont appréhendés au moment $\mathrm{t}$ de leur survenance, dans la séquentialité de leur enchaînement, en prenant en compte les effets de planification, d'anticipation et de rétroaction.

3. «[...] on définit rigoureusement la transitivité comme le mécanisme de phrase par lequel une entité [...], quelle qu'en soit la nature, adresse à l'après phrastique un appel de complément notionnel ou fonctionnel ».

4. On insiste sur le fait qu'il s'agit bien de «cette phrase » et d'un repère " possible », dans la mesure où les travaux de Sicot Domínguez (2010) ont montré que le signe espagnol /mas/ pouvait fonctionner sans repère; voir aussi Fortineau-Brémond (2012b). C'est le cas, par exemple, dans les phrases corrélatives du type más ... más. Étant donné l'objet d'étude de cet article, on ne prendra ici en compte que les cas de más de $\sim$ más que, et la remarque précédente n'affecte en rien la démonstration proposée. 
5. La présentation de l'exemple avec la flèche représentant le déroulé de la phrase dans le temps est typique de la chrono-analyse macchienne (2000); le choix des t1-t2-etc. est fonction des moments que l'on aura besoin de mentionner au cours de cet article. D'autres moments auraient bien sûr pu être pertinents dans le cadre d'une autre analyse.

6. En (2) : «Le PSOE (parti socialiste espagnol) maintient un avantage de plus de trois points sur le PP (parti populaire de droite espagnol) ». En (3) : «L'équipe en est arrivée à l'un des matchs les plus attendus de la saisons et se rend au colisée noir et blanc du Racing de Santander, l'une des équipes les plus fortes du groupe. En jeu, plus que trois points: un match pour reprendre le chemin de la victoire, se rapprocher de la Coupe et continuer à lutter jusqu'à la fin pour les playoffs ». (notre traduction)

7. En (4): «Marie-Hélène ne sort pas, ne se coiffe pas, ne se lave pas pendant deux semaines encore. Elle pense qu'elle va mourir. Et elle a raison. Higinio met plus de temps que d'habitude à rentrer de la ville de Mexico, mais il rentre avec un enfant ». En (5): «Dans cette émission improvisée (plus que d'habitude), nous vous donnons quelques recommandations quant aux émissions à regarder sur Netflix, et nous parlons aussi un peu du manga du Dr. Stone ». (notre traduction)

8. En (6) : «Les voyages étaient réalisés dans des conditions chaque fois pire que la précédente. Alors, elle devint une enfant méchante et insolente, elle mentait plus qu'elle ne parlait ». En (7) : «Blanche se chargea des papiers modifiés, et la grande Elsa, que les adultes considéraient comme la plus sensée des deux et dont personne ne doutait, car Blanche mentait plus qu'elle ne parlait, essaya de convaincre ses parents de ne pas les laisser y aller ». (notre traduction)

9. «Las comparaciones son odiosas» («les comparaison sont détestables») est un dicton espagnol bien connu, cité dans de nombreuses œuvres dès La Célestine et le Quichotte.

10. Plusieurs séries de rencontres et de publications ont eu lieu dernièrement en ce sens ; voir les colloques SAISIE (1ère édition à Dijon en 2014, org. D. Bottineau \& Ph. Monneret ; 2e édition à la Sorbonne Nouvelle en 2015, org. D. Bottineau, É. Blestel \& G. Le Tallec-Lloret, dont les actes sont à paraître dans la Revue de sémantique et pragmatique), colloques LangEnact (1ère édition à Clermont-Ferrand en 2016, actes parus dans le $\mathrm{n}^{\circ} 1$ de la revue Signifiances, 2e édition à l'université du Sud-Danemark d'Odense en 2017), séminaires du laboratoire junior ERILIIS (Équipe de Recherches Interlangues : Interlocution, Incarnation, Signifiant) à l'université Rennes 2 et dans les universités partenaires (Dijon, Lille, Sorbonne-Nouvelle et Paris-Nord Villetaneuse), voir https://www.univ-rennes2.fr/erimit/eriliis.

11. "Blanche mentait plus qu'elle ne parlait » (notre traduction). La distinction existante entre cette phrase «Blanca mentía más que hablaba » et celle attendue par la règle précédemment énoncée «Blanca mentía más de lo que hablaba » pourra être reprise et éclaircie plus avant dans l'article.

12. En (8) : «Ceci est plus joli que je ne l'avais imaginé » vs. « Ceci est plus joli que ce que j'avais imaginé » (notre traduction). En (9) : «J'en ai apporté plus que le strict nécessaire » vs. «J'ai apporté plus que le strict nécessaire» (notre traduction). En (10): «Plus de soixante communications ont été soumises à discussion » vs. "Plus que soixante communications ont été soumises à discussion » (notre traduction).

13. «Velázquez a peint d'autres tableaux à part Les Ménines » vs. "Velázquez a peint plus de tableaux que Murillo». (notre traduction)

14. Sauf indication contraire dans le tableau, les dénominations utilisées sont empruntées à Sáez \& Sánchez López (2013: 31-35), qui reprennent eux-mêmes d'autres dénominations traditionnellement utilisées dans la littérature sur la question.

15. Entités, relations ou circonstants. On reprend ici une terminologie de Fuchs (2014).

16. «Ces comparaisons 'impropres', parfois nommées 'pseudo-comparaisons' dans la bibliographie, constituent ainsi un cas flagrant d'usurpation formelle' qui oblige le chercheur à 
proposer des critères distinctifs explicites qui permettent de les différencier des comparaisons 'proprement dites'» (notre traduction).

17. «Le más des constructions additives n'entretient d'autre rapport avec le más de comparaison que celui de pure homophonie ». (notre traduction)

18. La NGLE (45.12f) reconnaît l'ambiguité pour une phrase du type El problema es más político que legal ou Es más trabajador que inteligente, par exemple, et propose une syntaxe alternative qui permette de lever l'ambiguité ; ainsi, El problema es político más que legal ou Es trabajador más que inteligente.

19. « Au pied des rochers, il y avait quelques maisons mal construites, qui ressemblaient plus à des ruines qu'à des maisons ». (notre traduction)

20. On prend là un certain raccourci, car à cet instant, ce que pourrait très bien aussi être introducteur de tout autre chose que le repère attendu, ce dernier étant alors rejeté à plus loin encore (par exemple : ... que más parecían ruinas que se habían derrumbado...).

21. C'est aussi la position défendue, en des termes légèrement différents, par Delport (2004: 46) : «Là où la langue ne permet pas l'alternance de deux formes, il [le locuteur] est sommé par elle d'appréhender l'expérience de la façon dont la forme l'appréhende ; aucun choix ne lui est laissé, une représentation lui est prescrite et il ne sait donc pas 'ce qu'il dit'. C'est quand il a l'exercice d'une liberté, quand la langue lui permet de choisir entre plusieurs manières d'appréhender l'expérience qu'il peut comprendre à quelle vision du monde il fait correspondre son dire ».

22. «Il y a plus d'un français. Celui qui débarque en pays gaulois en tant que touriste a parfois la sensation de se retrouver en terres verbalement mouvantes. On épuise très vite son français appris à l'école dès lors qu'un Français se met à parler allègrement en argot ou en verlan ». (notre traduction; dans ce contexte conflictuel, il semble que "plus qu'un français » serait également possible)

23. «L'équipe en est arrivée à l'un des matchs les plus attendus de la saisons et se rend au colisée noir et blanc du Racing de Santander, l'une des équipes les plus fortes du groupe. En jeu, plus que trois points : un match pour reprendre le chemin de la victoire, se rapprocher de la Coupe et continuer à lutter jusqu'à la fin pour les playoffs ». (notre traduction)

24. Les auteurs font en outre remarquer que seule la construction en que, par son caractère dialogal conflictuel, accepterait l'ajout de sólo - Se discutieron más que sólo sesenta ponencias (où sólo, placé après que, est alors attribué à la source interlocutive) - ou de mentira - Mentira, se discutieron más que sesenta ponencias (Sáez \& Sánchez López 2013 : 71). A l’inverse, ?Mentira, se discutieron más de (sólo) sesenta ponencias paraît incongru.

25. Cette remarque concernant la dissociation prosodique de más et de que dans l'exemple (8b) serait sans doute plus difficile à imaginer dans les exemples (11) et (12), ce qui n'affecte en rien la démonstration proposée ; l'effet de dissociation interlocutive y est juste décliné autrement, dans des contextes qui ne supposent pas la reprise corrective d'un énoncé antérieur prononcé par l'allocutaire.

26. "Ces entourages discursifs où más peut acquérir à lui seul le sens de 'plus de choses' ». (notre traduction)

27. Selon l'expression de Gutiérrez Ordóñez (1994b : 63), « el emisor pretende informar a su destinatario de la situación referencial de magnitud con relación a un punto o norma que se supone conocido " («l'émetteur prétend informer son destinateur d'une certaine quantité référée en rapport à un point de repère ou à une norme supposé(e) connu(e) »; notre traduction, c'est nous qui soulignons).

28. "Quand il y en a plus d'un. Parfois, nous devons faire plusieurs choses que nous n'avons pas envie de faire, il y a plus d'un dragon à tuer et nous ne savons pas vraiment lequel tuer en premier. C'est simple: choisis le plus gros. Quand tu auras tué le plus gros, les autres te paraitront peu importants ou faciles à tuer ». (notre traduction) 
29. «Mettre des guillemets entre le nom de famille et le prénom (ou ses initiales) de l'auteur : on ne mettra que des virgules pour séparer les auteurs (s'il y en a plus d'un). Les initiales du prénom ne seront pas suivies de point, sauf s'il s'agit du dernier auteur, qui sera suivi du point pour le séparer du titre du livre ». (notre traduction)

30. On remarquera que les rôles de locuteur $(\alpha) \sim$ allocutaire $(\beta)$ doivent être ici compris comme de pures places théoriques, la place $\beta$ pouvant parfaitement s'avérer être occupée par le locuteur lui-même (sans reconstruire d'interlocution, la simple pensée Al final, compré más que tres libros serait alors conflictuelle vis-à-vis de mes propres projections et attentes).

31. Une troisième configuration est le RID (Rapport Interlocutif Direct), sur lequel on passe dans le cadre de ce texte, mais qui pourrait volontiers correspondre à l'absence de repère associé à más.

32. Ainsi, à propos de l'article, Chevalier écrit: « [l'article], c'est, en fait, l'image anticipée d'un être qui est mise en place. D’un être doté de ses seules propriétées formelles, générique et numérique. L'apparition, dans la suite, du substantif niño n'est plus dès lors que la déclaration de l'identité notionnelle de cet être, ce qui nous fait regarder l'article comme 'l'appel d'un nom à venir'» (1999: 71).

33. On passe sur les débats concernant la nature de que dans les structures comparatives. On l'aura compris : quelle que soit la catégorie attribuée par l'analyste, il s'agit toujours bel et bien d'un seul et même signifiant que. Par ailleurs, certains, sentant bien sans doute la pertinence de l'alternance mais ne pouvant se résoudre à abandonner les catégories traditionnelles, vont jusqu'à parler d'un que comme préposition pour sortir de ce dilemme (Ortega Santos 2013; Romero Cambrón 1998). Le dilemme n'en est plus un dès lors que l'on considère les signifiants dans leur pure matérialité gestuelle, en les épurant de toute catégorie, lesquelles ne sont que le fruit d'une analyse réflexive.

34. [d] et [t] sont considérés comme les allomorphes voisé et non voisé du même cognème $T$. Voir Bottineau (2000).

35. Toussaint (1983 : 49) décrit les sons du point de vue de l'articulation en première personne, relativement au « déplacement de la colonne d'air au moment de la phonation ». Il renverse ainsi la description habituelle des phonèmes; de sorte que, par exemple, "les voyelles dites antérieures sont les ultérieures des voyelles couramment appelées postérieures ou vélaires. »

36. «Ce n'est qu'un mot, Ophélie. Rien d'autre qu'un mot». (notre traduction)

37. «Vous comprenez, maintenant, le mot 'au secours' ? C'est un peu plus qu'un mot, c'est une déclaration de principes au regard du fait que, presque dix ans après l'intronisation démocratique, le pacte qui a permis à la Transition d'avoir lieu révèle toute la misère qu'il a fallu avaler, tout le fascisme latent, ancien et nouveau, installé dans l'appareil de l'État et dans la moralité gouvernante $»$. (notre traduction)

38. «Il est travailleur plus qu'intelligent». (notre traduction)

39. «Chivas, plus qu'un whisky... c'est une légende »,; «Les Misérables. Plus qu'une comédie musicale, une légende »; «Plus qu'un couteau, une légende. Victorinox, c'est un sceau qui fait honneur à la culture et aux valeurs de la Suisse ». (notre traduction)

40. «Elle est très belle, votre mère. / Non, ce n'est pas ma mère. / Une tante ? / Non, c'est ma belle-mère. / Ah ! Vous êtes marié. Évidemment, comme vous ne portez pas d'alliance... Enfin, on n'en porte plus, aujourd'hui. [...] Et, sa fille? Enfin, votre femme? Ah, pardon ! Voilà que je pose plus de questions qu'il ne faudrait. C'est un de mes défauts. Le manque d'habitude ». (notre traduction)

41. «En ce qui concerne la nécessité de retourner à la table avec un dataphone, il est possible qu'on finisse par appliquer un tarif dissuasif, parce que 'le dataphone pèse beaucoup plus lourd que l'addition, il est plus loin, et il faut une formation pour l'utiliser', affirment les serveurs ». (notre traduction) 
42. «Le 2e terme de la comparaison agit comme un standard auquel on attribue pragmatiquement la possession (généralement, dans une proportion assez haute) de la propriété mesurée [...]. C'est le savoir pragmatique qui permet de reconstruire ce degré de la propriété qui fonctionne, dans le cadre de la comparaison, comme comparant. [...] Le 2e terme représente un modèle à partir duquel il est possible de déterminer, grâce à notre savoir pragmatique, un certain degré de la propriété comparée" (notre traduction). Cette catégorie proposée par Romero Cambrón semble correspondre à ce qui a été référencé dans le tableau 1 sous le nom de comparaciones hiperbólicas.

43. "Jean a des élèves plus coquins que Zipi et Zape" (notre traduction). Zipi y Zape sont deux petits personnages créés par José Escobar, protagonistes d'une série de bandes dessinées éponymes très populaires, éditées en Espagne depuis la fin des années 40. Leurs prénoms sont une décomposition de l'onomatopée zipizape, que le DRAE définit comme suit : riña ruidosa o con golpes (querelle bruyante ou impliquant des coups).

44. En (25): "Quel plus grand malheur peut-il y avoir que celui qui attend que le temps le consume et que la mort l'achève?». En (26): «Mauvais renégat, bien pire que Judas / je ne connais personne qui voudrait prier pour toi ». En (27): «Vie qui donne plus de clarté que le soleil, plus de légèreté que le vent, [...] un tel règne d'un tel roi est désirable ». (notre traduction)

45. En (28) : «Cette période est aussi connue comme la 'Période Médiévale Chaude' et a été suivie, entre 1450 et 1890 , d'un 'Petit Âge de Glace' - ou 'Petite ère glacière' -, où la température moyenne globale était un demi degré à un degré plus basse qu'elle ne l'est aujourd'hui. Plusieurs explications ont été proposées pour ces oscillations climatiques sur une courte durée ». En (29) : « Eh bien dit à Clarisse d'oublier la 'femme' qu'on lui a appris à être et celle qu'on a voulu qu'elle soit. Qu'elle arrête un peu de se comporter en victime, voyons! / Si elle suit ton conseil, elle finira encore plus seule qu'elle ne l'est aujourd'hui... / C'est incroyable que tout se résume à un 'comment ne pas finir seule'». En (30) : « S'il est vrai que le vin est un peu plus complexe que ne l'est la bière, si bien que nos amis brasseurs ont eu plus de facilité à l'obtenir, il me semble qu'il y a des bières sans alcool pour lesquelles je ne mettrais pas ma main à couper concernant la présence ou non d'alcool» (notre traduction).

\section{ABSTRACTS}

The question of the distribution de / que as "standard markers » is debated in the literature on comparison in Spanish. In this study, we examine this question in the light of an interlocutive chronosyntax approach, which studies the contribution of signifiers to the dynamics of sensemaking by the effets they produce on the interprent at the moment of their occurrence. We propose de and que operate differently in the «receptive framework» of interlocution, and we then show how this receptive framework functions in the various discourse meanings that grammars identify for más de and más que.

La question de l'alternance ou de la répartition de / que en position de « marqueur du standard » est l'une des plus débattues de la littérature sur la comparaison en espagnol. Dans cette étude, on se propose de reprendre cette question à la lumière d'une approche en chronosyntaxe interlocutive, qui étudie la contribution des signifiants à la dynamique de construction du sens pour les effets qu'ils produisent sur l'interprétant à l'instant de leur survenance. On propose la formulation d'une contribution opératoire de de et de que en termes de "cadre réceptif» 
qu'installent ces signifiants dans la relation interlocutive, et on montre ensuite la façon dont ce cadre réceptif sous-tend les divers effets de sens que recensent les grammaires pour más de et más que.

INDEX

Mots-clés: comparaison, signifiant, relation interlocutive (TRI), chronosyntaxe, cognématique. Keywords: comparison, signifying, interlocution, chronosyntax, cognematics.

\section{AUTHOR}

MARINE POIRIER

Université de Lille - SHS \& EA 3427 ERIMIT, Université Rennes 2 